\title{
The eROSITA Final Equatorial-Depth Survey (eFEDS) \\ LOFAR view of brightest cluster galaxies and AGN feedback
}

\author{
T. Pasini ${ }^{1}$, M. Brüggen ${ }^{1}$, D. N. Hoang ${ }^{1}$, V. Ghirardini' ${ }^{2}$, E. Bulbul ${ }^{2}$, M. Klein ${ }^{3}$, A. Liu ${ }^{2}$, T. W. Shimwell ${ }^{4,5}$, M. J. \\ Hardcastle $^{6}$, W. L. Williams ${ }^{5}$, A. Botteon ${ }^{5}$, F. Gastaldello ${ }^{7}$, R. J. van Weeren ${ }^{5}$, A. Merloni ${ }^{2}$, F. de Gasperin ${ }^{1,8}$, Y. E. \\ Bahar $^{2}$, F. Pacaud ${ }^{9}$, and M. Ramos-Ceja ${ }^{2}$ \\ 1 Hamburger Sternwarte, Universität Hamburg, Gojenbergsweg 112, 21029 Hamburg, Germany \\ 2 Max Planck Institute for Extraterrestrial Physics, Giessenbachstrasse 1, 85748 Garching, Germany \\ 3 Faculty of Physics, Ludwig-Maximilians-Universität, Scheinerstr. 1, 81679, Munich, Germany \\ 4 ASTRON, The Netherlands Institute for Radio Astronomy, Postbus 2, 7990 AA Dwingeloo, The Netherlands \\ ${ }^{5}$ Leiden Observatory, Leiden University, P.O. Box 9513, 2300 RA Leiden, The Netherlands \\ 6 Centre for Astrophysics Research, School of Physics, Astronomy and Mathematics, University of Hertfordshire, College Lane, \\ Hat- field AL10 9AB, UK \\ 7 IASF - Milano, INAF, Via A. Corti 12, I-20133 Milano, Italy \\ 8 INAF - Istituto di Radioastronomia, via P. Gobetti 101, 40129, Bologna, Italy \\ 9 Argelander-Institut für Astronomie (AIfA), Universität Bonn, Auf dem Hügel 71, 53121 Bonn, Germany
}

June 29, 2021

\begin{abstract}
Context. During the performance verification phase of the SRG/eROSITA telescope, the eROSITA Final Equatorial-Depth Survey (eFEDS) has been carried out. It covers a $140 \mathrm{deg}^{2}$ field located at $126^{\circ}<$ R.A. $<146^{\circ}$ and $-3^{\circ}<$ Dec. $<+6^{\circ}$ with a nominal unvignetted exposure over the field of $2.2 \mathrm{ks}$. 542 candidate clusters and groups were detected in this field, down to a flux limit $F_{X} \sim 10^{-14} \mathrm{erg} \mathrm{s}^{-1} \mathrm{~cm}^{-2}$ in the $0.5-2 \mathrm{keV}$ band.

Aims. In order to understand radio-mode feedback in galaxy clusters, we study the radio emission of brightest cluster galaxies of eFEDS clusters and groups, and we relate it to the X-ray properties of the host cluster.

Methods. Using LOFAR we identify 227 radio galaxies hosted in the BCGs of the 542 galaxy clusters and groups detected in eFEDS. We treat non-detections as radio upper limits. We analyse the properties of radio galaxies, such as redshift and luminosity distribution, offset from the cluster centre, largest linear size and radio power. We study their relation to the intracluster medium of the host cluster. Results. We find that BCGs with radio-loud AGN are more likely to lie close to the cluster centre than radio-quiet BCGs. There is a clear relation between the cluster's X-ray luminosity and the $144 \mathrm{MHz}$ radio power of the BCG. Statistical tests indicate that this correlation is not produced by biases or selection effects in the radio band. We see no apparent link between largest linear size of the radio galaxy and the central density of the host cluster. Converting the radio luminosity to kinetic luminosity, we find that radiative losses of the intracluster medium are in an overall balance with the heating provided by the central AGN. Finally, we tentatively classify our objects into disturbed and relaxed based on different morphological parameters, and we show that the link between the AGN and the ICM apparently holds for both subsamples, regardless of the dynamical state of the cluster.
\end{abstract}

Key words. Galaxies: clusters: intracluster medium - Galaxies: clusters: general - X-rays: galaxies: clusters - Radio continuum: galaxies - Galaxies: groups: general

\section{Introduction}

Radio galaxies that sit at the centres of galaxy clusters and galaxy groups play an important role in regulating the temperature of the Intra-Cluster Medium (ICM) and Intra-Group Medium (IGrM). Radio-loud Active Galactic Nuclei (AGN) are usually hosted by Brightest Cluster Galaxies (BCG) and they quench the cooling of the hot $\left(\sim 10^{7} \mathrm{~K}\right)$ ICM through mechanical feedback (see e.g. reviews by McNamara \& Nulsen 2012; Gitti et al. 2012). Effects of AGN feedback are manifested in the form of X-ray cavities and ripples in the cluster atmosphere (e.g., McNamara et al. 2000; Bîrzan et al. 2004; Fabian et al. 2006; Markevitch \& Vikhlinin 2007; Gastaldello et al. 2009). Consequences are also observed in the thermodynamical properties of the ICM, such as the gas entropy distribution (e.g., Cavagnolo et al. 2009), or in the transport of high-metallicity gas from the cluster centre to the outskirts (e.g., Liu et al. 2019). This type of feedback is generally positive, in the sense that when the radiative losses of the ICM increase, the AGN counteracts this by heating the ICM. The more gas cools and fuels the Super-Massive Black Hole (SMBH) at the centre of the BCG, the higher the energy output that is able to quench the ICM radiative losses and establish what is commonly known as $A G N$ feedback loop (see review from Gaspari et al. 2020). AGN feedback has been observed in systems ranging from isolated elliptical galaxies (Croton et al. 2006; Sijacki et al. 2015; O'Sullivan et al. 2011a) to massive clusters where it prevents the formation of cooling flows (McDonald et al. 2019; Ehlert et al. 2011; Pasini et al. 2021b). Most of the AGN associated with BCGs are in the so-called 'radio-mode' or 'maintenance-mode' (to distinguish it from the radiatively dominated quasar-mode feedback), where the accretion rate is modest and the feedback is mediated via 
mechanical work from powerful jets. A scaling relation between cavity power and radio luminosity, spanning over seven orders of magnitude in radio and jet power, has been observed in nearby systems (Bîrzan et al. 2004; Merloni \& Heinz 2007; Bîrzan et al. 2008; Cavagnolo et al. 2010; O'Sullivan et al. 2011b; Heckman \& Best 2014).

It has been pointed out that AGN feedback may operate differently in galaxy groups, where the gravitational potential is shallower (Sun 2012). Here, less energetic AGN than in clusters can have a larger impact on the IGrM (Giodini et al. 2010), since outbursts are also capable of expelling cool gas from the central region (Alexander et al. 2010; Morganti et al. 2013). As a result, AGN feedback may break the self-similarity between galaxy clusters and groups, especially in terms of their baryonic properties (Jetha et al. 2007). Hence, galaxy groups may be particularly interesting to study AGN feedback because their different environment should be reflected in the properties of the central AGN (e.g., Giacintucci et al. 2011).

Von Der Linden et al. (2007) found that Brightest Group Galaxies (BGGs) and BCGs lie on a different Fundamental Plane - in terms of velocity dispersion, effective radius and average surface brightness - and have experienced star formation for a shorter time than non-BCGs ${ }^{1}$. In the companion paper by Best et al. (2007), they also argued that BCGs are more likely to host radio-loud AGN than satellites of the same mass (cluster-hosted and not), but are less likely to host an optical AGN. These differences are particularly pertinent for BGGs. Main et al. (2017) studied the relation betweeen AGN feedback and central (at $0.004 R_{500}$ ) cooling time in a sample of 45 galaxy clusters. They find a clear correlation between AGN power and halo mass and $\mathrm{X}$-ray luminosity in clusters with a central cooling time of $<1$ Gyr.

X-ray observations of galaxy groups are more difficult than for galaxy clusters since groups have lower surface brightnesses and emit at lower temperatures, outside of the sweet spot of most X-ray observatories (see e.g., Willis et al. 2005). Still there has been some notable work on groups. Lovisari et al. (2015) have presented scaling relations in the group regime, while Johnson et al. (2009) and O'Sullivan et al. (2017) have classified their samples of groups into cool-core and non-cool-core. Kolokythas et al. (2018) have focused on central radio galaxies in the socalled Complete Local Volume Group Sample (CLoGS), and found that $\sim 92 \%$ of groups in their high-richness sample (26 objects) have dominant galaxies (BGGs) hosting radio sources. They also argued that radio galaxies showing jets are more common in bright groups, while radio non-detections are mostly found in X-ray faint systems. The same authors report, in the CLoGS low-richness sample (27 objects) studied in Kolokythas et al. (2019), a radio detection rate of $\sim 82 \%$ in the luminosity range $10^{20}-10^{25} \mathrm{~W} \mathrm{~Hz}^{-1}$ at $235 \mathrm{MHz}$. Malarecki et al. (2015) proposed that the lower densities in the IGrM, compared to the ICM, allows the lobes of group radio galaxies to expand to large distances. Werner et al. (2014) used Far InfraRed (FIR), optical, and X-ray data to study eight nearby giant elliptical galaxies, all central members of relatively low-mass groups. They find evidence that cold gas in those centrals galaxies is produced mostly by cooling from the hot phase and that this cool gas fuels outbursts of the AGN. Dunn et al. (2010) investigated a statistically complete sample of 18 nearby massive galaxies with X-ray and radio coverage, finding that 10 of them exhibit extended radio emission, with 9 also showing hints of interplay with the surrounding hot gas.

\footnotetext{
${ }^{1}$ hereafter we will refer to them as satellites for more clarity.
}

Mittal et al. (2009) determined that all cool-core clusters in a complete sample of $\sim 60$ clusters show a central radio galaxy, while only half of non-cool core clusters have one. Interestingly, when extending this study to galaxy groups, the trend becomes much weaker (Bharadwaj et al. 2014, 2015). A similar result was recently discussed in Pasini et al. (2020) (hereafter P20). In this paper, the authors studied a sample of $247 \mathrm{X}$-ray detected galaxy groups in the COSMOS field, matching them to radio galaxies detected in the VLA-COSMOS Deep Survey (Schinnerer et al. 2010) and in the COSMOS MeerKAT survey (MIGHTEE, Jarvis et al. 2016). They found that more than $70 \%$ of their radio galaxies are not hosted in BGGs, while in clusters $\sim 85 \%$ of central radio galaxies are associated with BCGs. They also discuss a correlation between the X-ray luminosity of groups and the radio power from the central radio galaxy since more massive groups seem to host more powerful sources. Indeed, Pasini et al. (2021a) recently showed that, in their sample of groups, BGGs showing powerful radio emission are always found within 0.2 $R_{\text {vir }} \sim 0.3 R_{200}$ from the centre.

The extended ROentgen Survey with an Imaging Telescope Array (eROSITA) onboard the Spectrum-Roentgen-Gamma (SRG) mission (Predehl et al. 2021) was launched on July 13, 2019. The large effective area $\left(1365 \mathrm{~cm}^{2}\right.$ at $\left.1 \mathrm{keV}\right)$, large field of view (FoV, 1 deg diameter), good spatial resolution (half energy width of $26^{\prime \prime}$ averaged over the FoV at $1.49 \mathrm{keV}, 16^{\prime \prime}$ onaxis) and spectral resolution $(\sim 80 \mathrm{eV}$ full width half maximum at $1 \mathrm{keV}$ ) of eROSITA allow unique survey science capabilities by scanning large areas of the X-ray sky quickly and efficiently (Merloni et al. 2012). Thus, eROSITA is detecting a large number of previously undetected groups and clusters, most of them with low surface brightnesses and at low redshifts, even though the confirmation of these groups in the optical is challenging for $z<0.1-0.2$.

In this work, we exploit the results of the eROSITA Final Equatorial-Depth Survey (eFEDS), a mini-survey designed to demonstrate the science capabilities of eROSITA. We study the radio galaxies observed in cluster centres at a frequency of 144 $\mathrm{MHz}$ by the LOw Frequency ARray (LOFAR, van Haarlem et al. 2013) in order to investigate their relation to their host clusters. This paper is structured as follows: in Sec. 2 we give a detailed description of how we build the sample. In Sec. 3 we show the results, compare them to previous work and analyse the implications for AGN feedback. Finally, in Sec. 4 we summarise our results. Throughout this paper, we assume a standard $\Lambda \mathrm{CDM}$ cosmology with $\mathrm{H}_{0}=70 \mathrm{~km} \mathrm{~s}^{-1} \mathrm{Mpc}^{-1}, \Omega_{\Lambda}=0.7$ and $\Omega_{\mathrm{M}}=1-\Omega_{\Lambda}=0.3$.

\section{The sample}

\subsection{The eROSITA observation of eFEDS and the cluster catalog}

eFEDS covers a $140 \mathrm{deg}^{2}$ field located in an equatorial region, with R.A. from $\sim 126$ to $\sim 146 \mathrm{deg}$, and declination from $\sim-3$ to $\sim 6 \mathrm{deg}$. This field was uniformly scanned by eROSITA during the Performance Verification phase resulting in a nominal exposure of about $2.2 \mathrm{ks}$ (unvignetted) over the field, which is similar in depth to the final exposure that will be reached in 4 years in equatorial fields in the eROSITA All-sky survey (Liu et al. 2021).

The eFEDS data were acquired by eROSITA over 4 days, between November 4 and 7, 2019. These data were processed by the eROSITA Standard Analysis Software System (eSASS, Brunner et al. 2021). We refer to Ghirardini et al. (2021, here- 
after G21) for further details on the data processing. The source detection was performed using the tool erbox in eSASS, on the merged $0.2-2.3 \mathrm{keV}$ image of all seven eROSITA Telescope Modules (TMs). erbox is a modified sliding box algorithm, which searches for sources in the input image that are brighter than the expected background fluctuation at a given image position. For each candidate source, the detection likelihood and the extent likelihood are determined by fitting the image with the source model, which is a $\beta$-model convolved with the calibrated PSF. Sources with extension too broad to be fitted by the PSF have a larger extent likelihood. For further details on the source detection procedure we refer to Brunner et al. (2021). We detect 542 candidate clusters over the full field (Liu et al. 2021). This corresponds to a source density of $\sim 4$ clusters per square degree at the equatorial depth. Photometric redshifts are obtained through the Multi-Component Matched Filter (MCMF) cluster confirmation tool (Klein et al. 2018). Optical data from the Hyper Suprime-Cam Subaru Strategic Program (HSC-SSP, Aihara et al. 2018) and from the DESI Legacy Survey (LS, Dey et al. 2019) were exploited. We refer to Klein et al. (2021) and Liu et al. (2021) for further details. Spectroscopic redshifts derived from 2MRS (Huchra et al. 2012), SDSS (Blanton et al. 2017) or GAMA (Driver et al. 2009) are used when available (296 out of 542 clusters). For each cluster, a massive red-sequence galaxy near the X-ray emission peak is selected as the BCG, following Klein et al. (2021).

The sample of clusters can be expected to be contaminated by spurious sources, or misclassified AGN, at a level of $\sim 19.7 \%$ (see Liu et al. 2021). Cluster contamination is therefore taken into account through the parameter $f_{\text {cont }, i}$ (Klein et al. 2019), which is defined as:

$f_{\text {cont }, i}=\frac{\int_{\lambda_{i}}^{\infty} f_{\text {rand }}\left(\lambda, z_{i}\right) d \lambda}{\int_{\lambda_{i}}^{\infty} f_{\text {obs }}\left(\lambda, z_{i}\right) d \lambda}$,

where $f_{\text {rand, } \mathrm{z}}$ is the richness distribution of random positions at the cluster candidate redshift $z_{i}, f_{\text {obs }}\left(\lambda, z_{i}\right)$ the richness distribution of true candidates and $\lambda_{i}$ the richness of the cluster candidate. The estimator $f_{\text {cont }, i}$ is correlated with the probability of a source being a chance superposition. Applying a given cut to this parameter allows us to reduce the initial contamination of the cluster sample. Assuming independence between contaminants in the X-ray sample, the fractional contamination of the sample is simply the product of the initial fractional contamination of the $\mathrm{X}$-ray sample and the applied cut in $f_{\text {cont }}$. For example, applying a cut in $f_{\text {cont, } i}<0.3$ results in a sample of about $88 \%(477 / 542)$ of the eFEDS extended sources being confirmed as galaxy clusters. Assuming an initial contamination of the X-ray sample of $20 \%$ (Klein et al. 2021), this $f_{\text {cont }}$ selected sample is expected to contain $\sim 6 \%$ contamination. Subsequent tests described in Klein et al. (2021) confirm the expected amount of contamination to be $6 \pm 3 \%$. For more details about the X-ray catalog we refer to Liu et al. (2021), while further details on the optical confirmation and contamination can be found in Klein et al. (2021).

\subsection{The LOFAR observations of eFEDS and the radio source catalog}

The eFEDS field was observed with the LOFAR High Band Antennae (HBA) for a total of 184 hours (including simultaneous observations by two LOFAR pointings) between February 24, 2016 and May 27, 2020 by projects LC13_029 (100 hours), LT5_007 (32 hours), LT10_010 (44 hours), and LT14_004 (8
Table 1. LOFAR HBA observations of the eFEDS field

\begin{tabular}{lc}
\hline \hline Telescope & LOFAR \\
\hline Project & LC13_029, LT5_007, \\
Mode & LT10_010, LT14_004 \\
Pointing & HBA_DUAL_INNER \\
& eFEDS_128, eFEDS_131, \\
& eFEDS_134, eFEDS_136, \\
& eFEDS_139, eFEDS_142 \\
& P129+02, P132+02, \\
& P134+02, P137+02, \\
& P139+02, P126+02 \\
& G09_A, G09_B, \\
Calibrator & G09_C, G09_D \\
Frequency (usable, MHz) & 3C 196, 3C 295 \\
Central frequency (MHz) & $120-168$ \\
Number of subbands (SB) & 144 \\
Bandwidth per SB (kHz) & 241 \\
Channels per SB & 195.3 \\
On-source time (hr) & 16 \\
Integration time (s) & $184^{a}$ \\
Frequency resolution $(\mathrm{kHz})$ & 1 \\
Correlations & 12.2 \\
Number of stations & XX, XY, YX, YY \\
& 73-75 (48 split core, \\
\hline \hline Notes: ${ }^{a}:$ calculated from the total duration on all pointings, \\
including simultaneous observations with two LOFAR beam; ${ }^{b}:$ \\
International stations are not used in this study. \\
14 remote, 9-13 international \\
\hline$b$
\end{tabular}

hours). The eFEDS field is entirely covered by six pointings of LC13_029 that are separated by 2.7 degree in a row. The LT5_007 observations that are centred on the GAMA 09 field cover the central region of the eFEDS field with four pointings separated by 2.4 degree. LT10_010 and LT14_004, as part of the LOFAR Two-meter Sky Survey (LoTSS; Shimwell et al. 2017, 2019), are positioned on the LoTSS grid where pointings are typically 2.58 degree apart. We present a layout of the LOFAR observations of the eFEDS field in Fig. 1. The setup for all observations is described in detail in Shimwell et al. (2017, 2019). The observing frequency is from $120 \mathrm{MHz}$ to $187 \mathrm{MHz}$, but we remove the data above $168 \mathrm{MHz}$ where the signal is highly contaminated by RFI. Each pointing was performed by multiple chunks of 2 or 4 hours when the field is at high elevation (i.e. an average elevation of 35 degree). Bright radio sources 3C 196 and/or 3C 295 were observed for 10 minutes each before and after the observations of the target fields and are used as primary calibrators. Details of the observations are given in Table 1.

We adopt the standard calibration procedure that has been developed for LoTSS (Shimwell et al. 2017, 2019). The calibration aims to correct for the direction-independent and directiondependent effects (e.g. ionosphere and beam model errors) which need to be corrected for high-fidelty imaging with the LOFAR HBA. The data for each pointing were separately processed with PREFACTOR ${ }^{2}$ (van Weeren et al. 2016; Williams et al. 2016; de Gasperin et al. 2019) and DDF-pipeline ${ }^{3}$ (Tasse 2014a,b; Smirnov \& Tasse 2015; Tasse et al. 2018; Tasse et al. 2021). In detail, the processing was identical to that described by Tasse et al. (2021) with one exception: in order to deal with the effect of sources outside the target $8 \times 8$ degree field but still covered by

\footnotetext{
2 https://github.com/lofar-astron/prefactor

3 https://github.com/mhardcastle/ddf-pipeline
} 


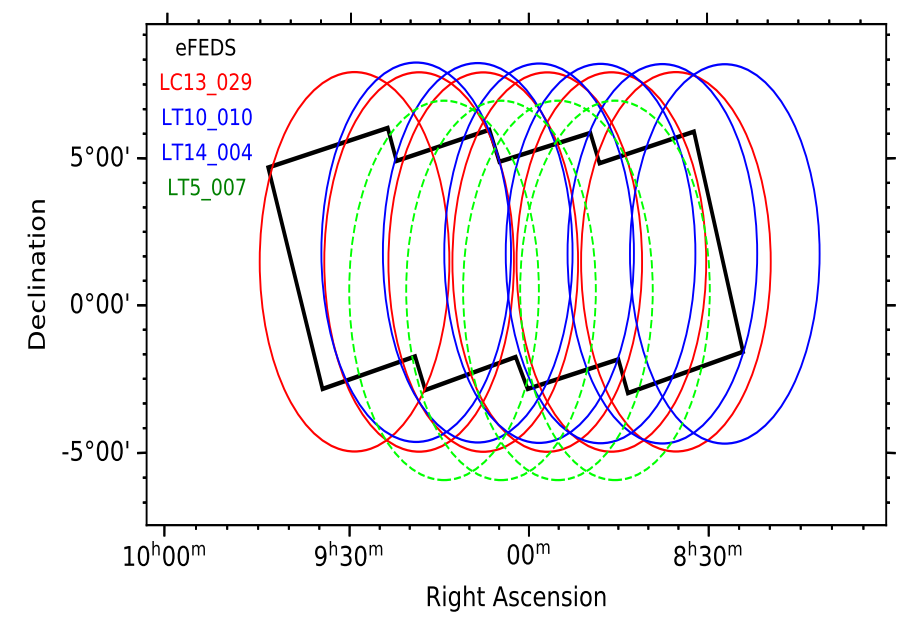

Fig. 1. LOFAR observations of the eFEDS field, shown in the black region. The elliptical lines show the LOFAR pointing locations in the projects LC13_029 (red), LT5_007 (green), LT10_010 (blue), and LT14_004 (blue). The major and minor axes of the ellipse (i.e. 4.0 degree and 6.7 degree) are the FWHM of the LOFAR station beam along the RA and Dec axes, respectively.

the very N-S elongated LOFAR primary beam, the first step of the pipeline for each image was to make a very large $(27 \times 27 \mathrm{de}-$ gree) image of the whole primary beam and subtract off sources detected by DDFacet that appeared in this image but lay outside the target field.

The pipeline produces high-resolution $\left(<9^{\prime \prime}\right)$ images for each pointing with an rms noise of $\approx 170 \mu \mathrm{Jy} \mathrm{beam}^{-1}$ in the pointing centre and $\approx 335 \mu \mathrm{Jy}$ beam $^{-1}$ in the regions $2.5 \mathrm{de}$ gree from the pointing centres. Given the large LOFAR station beam (i.e. FWHM of 4 degree in an E-W direction at the central frequency of $144 \mathrm{MHz}$ ), the separation of 2.4-2.7 degree between the pointings leads to a significant overlap between the images. To increase the fidelity of the images, we convolved the images to a common resolution of $8^{\prime \prime} \times 9^{\prime \prime}$ and made a mosaic of the entire eFEDS field in the manner described by Shimwell et al. (2019), reprojecting each image onto a 50,000 $\times 27,000$ pixel image with 1.5 -arcsec pixels centred on $\mathrm{RA}=9 \mathrm{~h}, \mathrm{Dec}=1$ degree and then combining the reprojected images weighting by the local image noise at each pixel, taking account of the primary station beam. No astrometric blanking was carried out in the mosaicing and each image was corrected before mosaicing to the flux scale of Roger et al. (1973) in the manner described by Hardcastle et al. (2021). The noise in the resulting mosaic is non-uniform but reduces to $\approx 135 \mu \mathrm{Jy}$ beam $^{-1}$ in the central parts of the image.

To produce a catalog of radio sources, we performed source detection on the high-resolution $\left(8^{\prime \prime} \times 9^{\prime \prime}\right)$ mosaic of the eFEDS field with the Python Blob Detector and Source Finder (PyBDSF ${ }^{4}$; Mohan \& Rafferty 2015). Sources were detected with a peak detection threshold of $5 \sigma$ (thresh_pix=5) and an island threshold of $4 \sigma$ (thresh_isl=4) that limits the boundary for the source fitting. Here the local noise rms, $\sigma$, is calculated using a box of $(150 \times 150)$ pixels $^{2}$ that slides across the mosaic with a step of 15 pixels. Around bright sources, typically compact, where the pixel values are higher than $150 \sigma$ (adaptive_thresh $=150)$, we used a smaller box of $(60 \times$ $60)$ pixels $^{2}$ and a sliding step of 15 pixels. The smaller box is more accurate for the estimate of the high noise rms around

\footnotetext{
${ }^{4}$ https://github.com/lofar-astron/PyBDSF
}

bright sources. The source detection produces a catalog of 45,207 sources, most of which (99.6 percent) have $144 \mathrm{MHz}$ flux densities below $1 \mathrm{Jy}$.

The mosaic that is made with the standard procedure described above typically has a flux density uncertainty of 10 percent. However, to further check the flux scale in the eFEDS mosaic we compare the integrated flux densities of the LOFAR detected sources with those in the TGSS-ADR1 (TIFR GMRT Sky Survey - Alternative Data Release 1, Intema et al. 2017) $150 \mathrm{MHz}$ data, which has similar central frequency. The LOFAR mosaic was smoothed to the resolution of the TGSS-ADR1 (i.e. $25^{\prime \prime}$ ) and regridded to match the spatial dimensions of the TGSS-ADR1 image. Radio sources in the LOFAR and TGSSADR1 25" images are detected with PyBDSF in an identical manner as done for the LOFAR $8^{\prime \prime} \times 9^{\prime \prime}$ mosaic above. There are 4,585 sources detected with both LOFAR and TGSS-ADR 1 observations. Sixty percent of these sources (i.e. 2,695) are modeled by a single Gaussian and are used for the flux scale comparison. Since the observing frequencies for the LOFAR and TGSS-ADR1 data are different, we rescaled the flux densities of the TGSS-ADR1 sources to match those at the frequency of the LOFAR data (144 MHz) by assuming a common spectral index of 0.8 (see Sec. 2.3 for a definition). We performed a linear fit to the LOFAR and TGSS-ADR 1 scaled flux densities, weighting by the LOFAR flux densities, and obtained a relation $\log _{10}\left(S_{\text {LOFAR }}\right)=0.92 \times \log _{10}\left(S_{\text {TGSS-ADR } 1 ; \text { scaled }}\right)+0.03$ [Jy]. The integrated flux densities of the radio sources in the LOFAR cata$\log$ is $\sim 10$ percent higher than those in the TGSS-ADR1 catalog. In this paper, we assume an uncertainty of 20 percent for the integrated flux densities of the LOFAR detected sources. In Fig. 2, we present a scatter plot between the flux densities of the LOFAR and TGSS-ADR1 detected sources. The LOFAR detected sources, especially the faint ones, have higher flux densities than those found with the TGSS-ADR1 observations.

Following Shimwell et al. (2019), we checked the astrometry of the sources detected with PyBDSF in the LOFAR $8^{\prime \prime} \times$ $9^{\prime \prime}$ mosaic by comparing their locations with those of their FIRST $1.4 \mathrm{GHz}$ counterparts. We use the FIRST survey due to its high astrometric accuracy of $0.1^{\prime \prime}$ compared to the absolute radio reference frame (White et al. 1997) and the comparable spatial resolution of both surveys (i.e. $5^{\prime \prime} \times 5^{\prime \prime}$ for FIRST and $8^{\prime \prime} \times 9^{\prime \prime}$ for LOFAR). We cross-matched the sources within a radius of $9^{\prime \prime}$ in the LOFAR and FIRST catalogs and found 10,709 common sources, of which 6,601 are single-Gaussian LOFAR sources. We calculate the offsets in RA and Dec for these singleGaussian sources and present them in Fig. 2. The histograms of the RA and Dec offsets are fitted with a Gaussian whose location and standard deviation are defined as the systematic offsets and total astrometric uncertainty. There are systematic offsets of $0.13^{\prime \prime}$ and $0.04^{\prime \prime}$ in RA and Dec, respectively. The standard deviations of the offsets in RA and Dec are $0.70^{\prime \prime}$ and $0.82^{\prime \prime}$, respectively. When comparing to the offsets between FIRST and LoTSS sources (Shimwell et al. 2019), our results on the RA and Dec offsets are a factor of two to seven higher, and the standard deviations are a factor of two to three higher. These are likely due to the lower declination of the eFEDS field, as compared with the declination of $\approx 50^{\circ}$ of the LoTSS-DR1 field, that results in a larger elongated beam and slightly more disturbed ionospheric conditions. However, the uncertainties are well within the resolution of the LOFAR observations (i.e. $8^{\prime \prime} \times 9^{\prime \prime}$ ). 

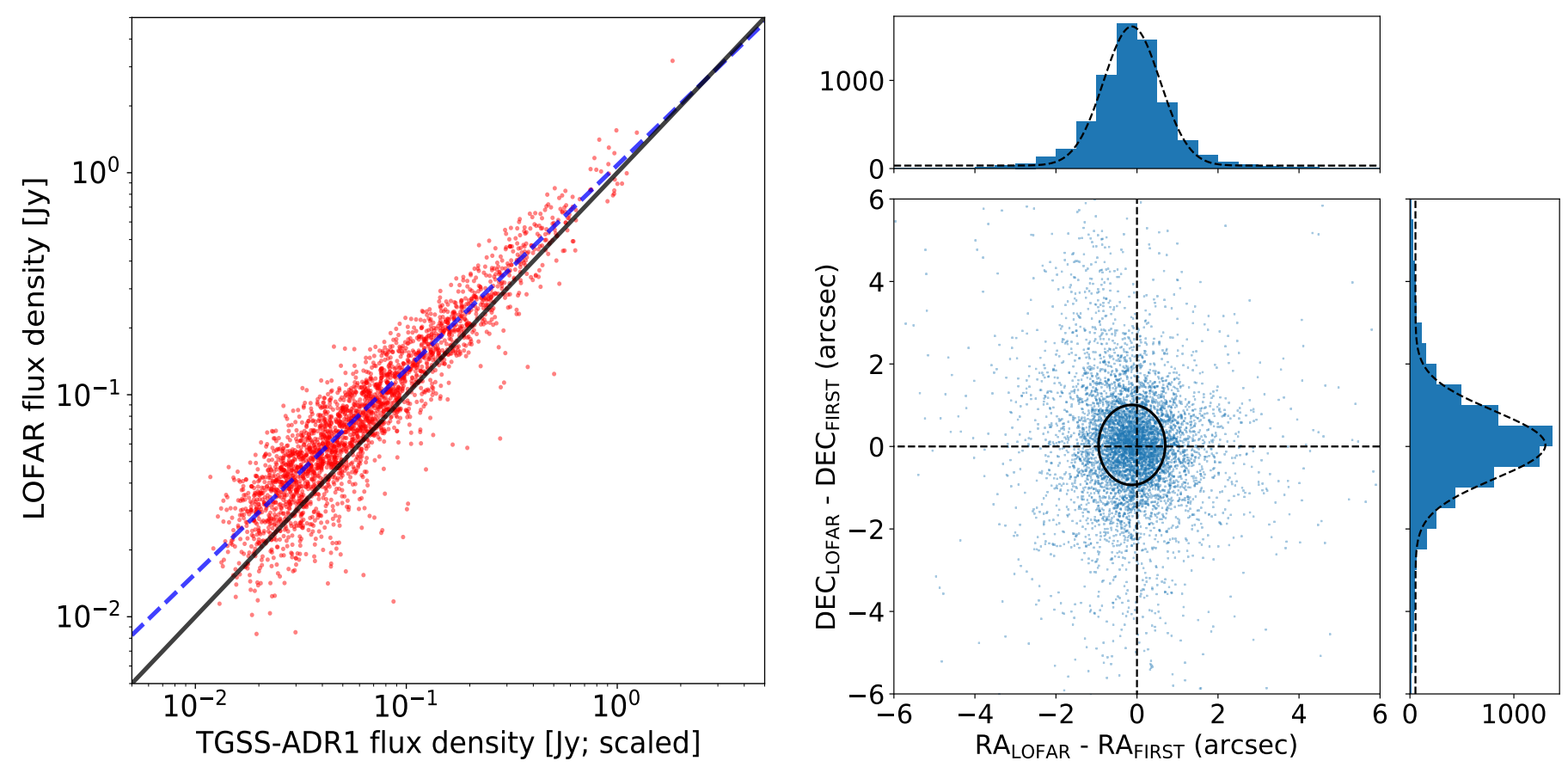

Fig. 2. Left: Scatter plot between the TGSS-ADR1 (scaled) and LOFAR flux densities for single-Gaussian sources. The dashed blue line is the best fit of the LOFAR and TGSS-ADR1 (scaled) flux densities, $\log _{10}\left(S_{\mathrm{LOFAR}}\right)=0.92 \times \log _{10}\left(S_{\mathrm{TGSS}-\mathrm{ADR} 1 \text {; scaled }}\right)+0.03$ [Jy]. The black solid line is a diagonal line with slope 1. Right: RA and Dec offsets for the LOFAR and FIRST detected single-Gaussian sources. The histograms of the offsets, including the best-fit Gaussian dashed lines, are plotted in the top and right panels, respectively. The ellipse shows the peak location (i.e. 0.13" to the left and 0.04" to the top of the centre point) and the FWHM (i.e. $0.70^{\prime \prime}$ and $0.82^{\prime \prime}$ in RA and Dec.) of the Gaussian functions that are obtained from the fitting of the RA and Dec offset histograms.

\subsection{Sample construction and properties}

The catalog of radio sources was cross-matched with the BCG positions (see Sec. 2.1) by setting a sky threshold $\sim 3 \theta$, with $\theta$ being the synthesised beam of the interferometric radio observation. The results were then manually inspected to check for the presence of false positives (i.e. radio sources incorrectly associated with an optical BCG) or false negatives (i.e. radio emission lying at more than $3 \theta$ from the $\mathrm{BCG}$, but with an obvious association to it). We find no wrong BCG-radio association, while two clusters were initially mistakenly classified as non-detections. To limit contamination, we applied the same cut $f_{\text {cont }, i}<0.3$ discussed in Sec. 2.1. According to Eq. 1, this implies that we are statistically allowing for $6 \%$ contamination. This value, albeit conservative, produces a relatively small impact on our results.

The final catalog contains a total of 227 clusters, with only $\sim 1 \%$ ( 3 out of 230 ) of objects lost to contamination. This is consistent with our expectations, since the cut we applied should result in a cluster catalog that is $\sim 99 \%$ complete (see Klein et al. 2021 and Liu et al. 2021). Out of the parent sample of 542 X-ray clusters, 312 did not match any of LOFAR radio sources. After applying the same contamination criteria, we were left with 248 clusters with no radio emission detected, losing $\sim 21 \%$ of the original sample. These were then treated as radio upper limits assuming a flux limit of $3 \sigma$, where $\sigma$ is the local $r m s$ noise of the LOFAR mosaic at the position of the cluster. The increase in the number of clusters lost to contamination with respect to detections is easy to explain, once it is considered that excluded objects are not real clusters, but mostly contaminants (e.g. bright AGN). Therefore, it is less likely to find a radio counterpart. Again, we refer to Klein et al. (2021) and Liu et al. (2021) for further details.
Nevertheless, not every cluster/group, in reality, hosts radio galaxies. In fact, some groups only contain a few $(<10)$ galaxies, and only $\sim 1 \%$ of all observed galaxies are active (Padovani et al. 2017). This fraction should also be significantly higher in overdense environments such as clusters. Sabater et al. (2019) found that $100 \%$ of their sample of AGN in massive galaxies $\left(>10^{11}\right.$ $\mathrm{M}_{\odot}$ ) are always switched on above a $144 \mathrm{MHz}$ luminosity of $10^{21} \mathrm{~W} \mathrm{~Hz}^{-1}$. In fact, it has been observed that there is a strong link between the presence of radio AGN activity and the host galaxy mass (Best et al. 2005; Sabater et al. 2013). As already discussed in Sec. 1, Kolokythas et al. $(2018,2019)$ report rates at $235 \mathrm{MHz}$ of $92 \%$ and $82 \%$ for their sample of 26 and 27 galaxy groups, respectively. P20 report a detection rate for COSMOS groups of $\sim 70 \%$, with $r m s \sim 12 \mu \mathrm{Jy}^{\text {beam }^{-1}}$. Here, the same fraction is only $48 \%$ (given the cut we applied for contamination). This is likely due to the lower Signal-to-Noise ratio $(\mathrm{S} / \mathrm{N})$ of LOFAR eFEDS with respect to the single-target observations used to build CLoGS, while P20 exploited the VLA-COSMOS Deep Survey. Furthermore, CLoGS was built with low-redshift $(z<0.02)$ groups, while our sample reaches $z \sim 1.3$.

The luminosity of all the radio sources, including upper limits, was estimated as:

$L_{144 \mathrm{MHz}}=S_{144} 4 \pi D_{\mathrm{L}}^{2}(1+z)^{\alpha-1}$,

where $\mathrm{S}_{144}$ is the flux density at $144 \mathrm{MHz}, \mathrm{D}_{\mathrm{L}}$ is the luminosity distance at redshift $z$ and $\alpha$ is the spectral index $S_{v} \propto v^{-\alpha}$, assumed $\sim 0.8$ for all radio galaxies since we are at low frequency and most sources show a relatively extended morphology, rather than being compact and point-like as usually observed at higher frequency.

The left panel of Fig. 3 presents the redshift distribution for the sample, classified into detections and radio upper limits. The detection and non-detection distributions look similar up to $z \sim$ 

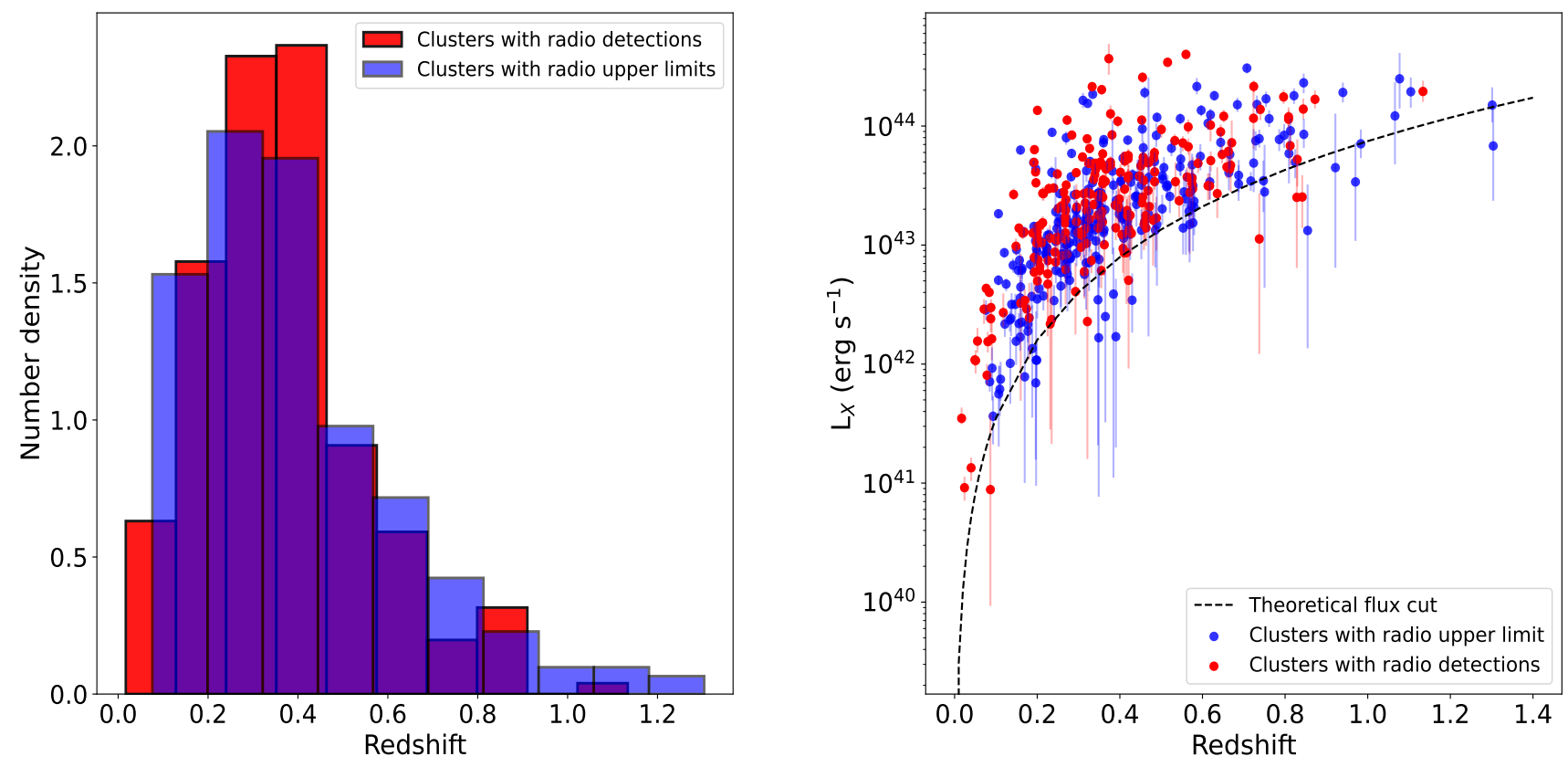

Fig. 3. Left: Histogram showing the redshift distribution of the sample, classified into radio detections (red) and upper limits (blue). Right: $L_{X, 500}$ vs. redshift for the sample. The dashed line denotes the theoretical flux cut of the eROSITA observation.

0.9. The highest- $z$ detection is at $z \sim 1.1$, while there is one radio upper limit at $z \sim 1.3$. The right panel shows $L_{X, 500 \mathrm{kpc}}$ vs. redshift, with the same classification, with $L_{X, 500 \mathrm{kpc}}$ being the 0.5 $2.0 \mathrm{keV}$ luminosity measured within a $500 \mathrm{kpc}$ radius. The flux sensitivity is $F_{X}=1.5 \times 10^{-14} \mathrm{erg} \mathrm{s}^{-1} \mathrm{~cm}^{-2}$. Further details on the eROSITA selection function and completeness can be found in Liu et al. (2021).

\section{Analysis and discussion}

\subsection{X-ray and radio luminosity distributions}

In Fig. 4 we show the X-ray and radio luminosity distributions. The X-ray distribution, in the left panel, spans the range from $L_{X, 500 \mathrm{kpc}} \sim 10^{41} \mathrm{erg} \mathrm{s}^{-1}$ to $4 \times 10^{44} \mathrm{erg} \mathrm{s}^{-1}$ for objects with radio detections, while the range for clusters with upper limits is slightly narrower, reaching $3 \times 10^{44} \mathrm{erg} \mathrm{s}^{-1}$. Due to the high sensitivity of eROSITA, we are able to reach lower luminosities than the existing X-ray samples of clusters and groups. The BCS sample, compiled with ROSAT (Ebeling et al. 1997), reaches $L_{X} \sim 10^{42} \mathrm{erg} \mathrm{s}^{-1}$, similarly to the REFLEX II catalog (Böhringer et al. 2014). On the other hand, our upper range is lower than both the BCS and the REFLEX II, which go well beyond $L_{X} \sim 10^{45} \mathrm{erg} \mathrm{s}^{-1}$, since our sample comes from a relatively small field in the sky. The forthcoming eROSITA all-sky survey (eRASS, Bulbul et al. in prep.) will observe a large number of clusters and groups, allowing to extend our analysis to higher luminosities.

The radio luminosity distribution at $144 \mathrm{MHz}$, in the right panel, ranges from $L_{144 \mathrm{MHz}} \sim 10^{29} \mathrm{erg} \mathrm{s}^{-1} \mathrm{~Hz}^{-1}$ to $\sim 10^{34}$ $\mathrm{erg} \mathrm{s}^{-1} \mathrm{~Hz}^{-1}$. Given the assumption on the spectral index made above, the upper range of luminosities at $144 \mathrm{MHz}$ corresponds to $L_{1.4 \mathrm{GHz}} \sim 1.6 \times 10^{33} \mathrm{erg} \mathrm{s}^{-1} \mathrm{~Hz}^{-1}$. This is lower than other samples that have recently been studied at this frequency. The catalog of $1.4 \mathrm{GHz}$ radio sources in galaxy groups analysed in
P20 reaches $L_{1.4 \mathrm{GHz}} \sim 10^{34} \mathrm{erg} \mathrm{s}^{-1} \mathrm{~Hz}^{-1}$, similarly to the sample of BCG radio galaxies by Hogan et al. (2015). Finally, we note that the sample studied at $235 \mathrm{MHz}$ by Kolokythas et al. (2018, 2019) ranges from $L_{235 \mathrm{MHz}} \sim 10^{27}$ to $10^{32} \mathrm{erg} \mathrm{s}^{-1} \mathrm{~Hz}^{-1}$. Converting from $144 \mathrm{MHz}$ luminosity, eFEDS radio galaxies span from $L_{235 \mathrm{MHz}} \sim 6.8 \times 10^{28}$ to $6.7 \times 10^{33} \mathrm{erg} \mathrm{s}^{-1} \mathrm{~Hz}^{-1}$. Therefore, our sample extends to higher radio powers, but does not go as deep as CLoGS. Nevertheless, it consists of 227 clusters and groups, compared to the 53 groups that belong to CLoGS.

\subsection{BCG offsets}

Fig. 5 shows the histogram of the BCG offset from the centre of the host cluster/group. The centre was estimated by fitting a two-dimensional $\beta$-model (Cavaliere $\&$ Fusco-Femiano 1976) to the X-ray emission. Most BCGs with detected AGN radio emission lie within $\sim 50 \mathrm{kpc}$ from the cluster centre $(\sim 84 \%)$. For these clusters, the median value of the offset distribution is $\sim 15 \mathrm{kpc}$, with dispersion $\sim 30 \mathrm{kpc}$. At larger offsets it is easier to find BCGs that do not host a radio galaxy. For clusters with no radio detection, the median is $\sim 130 \mathrm{kpc}$ with dispersion $\sim 190 \mathrm{kpc}$.

Small offsets $(<50 \mathrm{kpc})$ are expected and found in most relaxed clusters since even a minor merger can induce sloshing and displace the X-ray emission peak from the BCG (e.g., Hamer et al. 2016; Pasini et al. 2019; Ubertosi et al. 2021; Pasini et al. 2021b). Large offsets (100-1000 kpc) are often an indication of a strongly disturbed cluster environment (Rossetti et al. 2016; De Propris et al. 2021, and references therein). The relation between BCGs, the triggering of the AGN and the offset from the cluster centre has been widely discussed and was recently studied in Pasini et al. (2021a). In that paper, the authors found that it is more common for more central BCGs to show radio-loud AGN since in these galaxies the accretion onto the central $\mathrm{BH}$ is boosted by the strong cooling in the cluster core. Similar results 

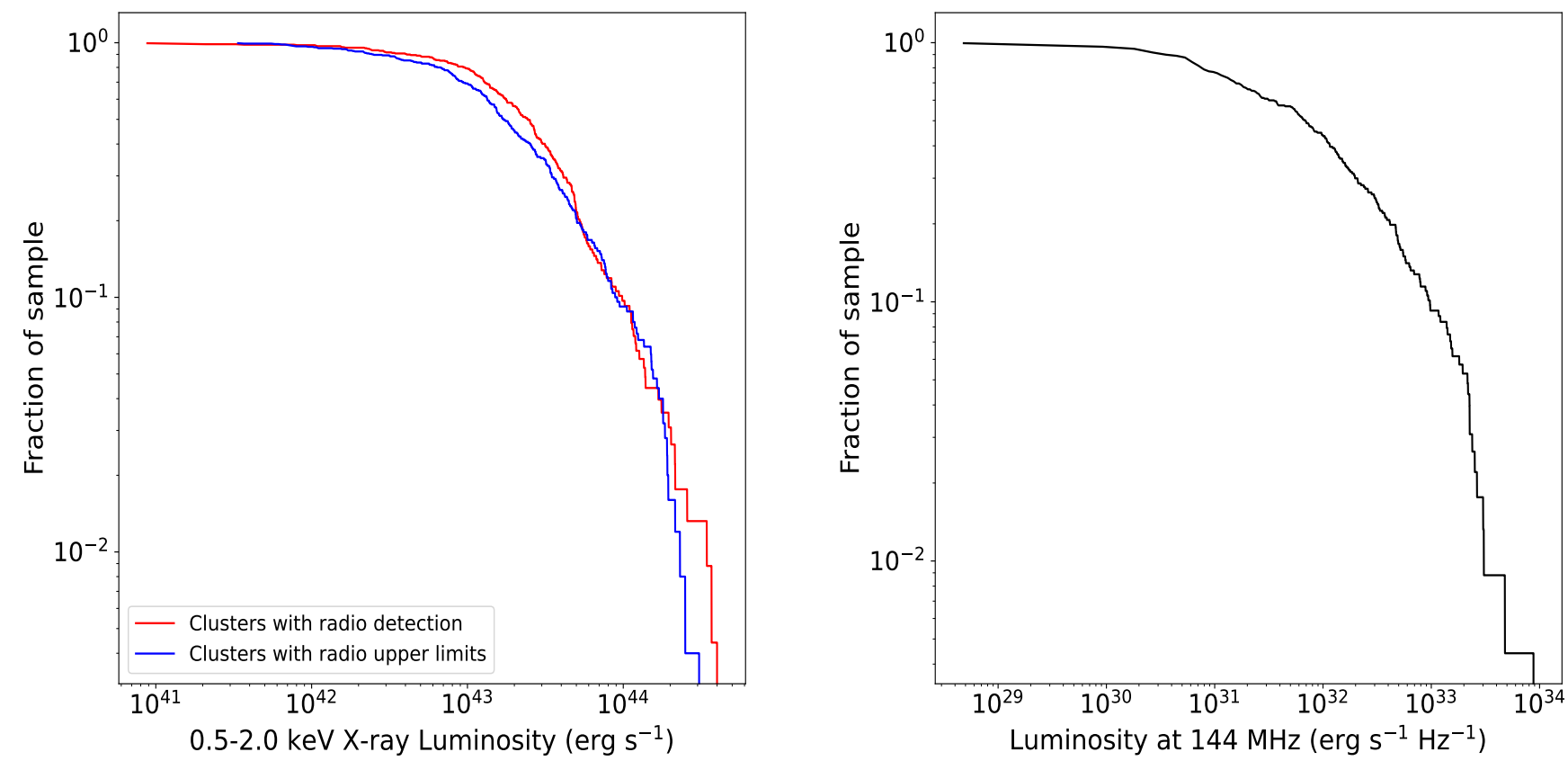

Fig. 4. Left: X-ray luminosity distribution for the parent sample of clusters and groups, divided into objects with (red) and without (blue) radio detection. Right: Radio luminosity distribution for clusters and groups with radio detection.

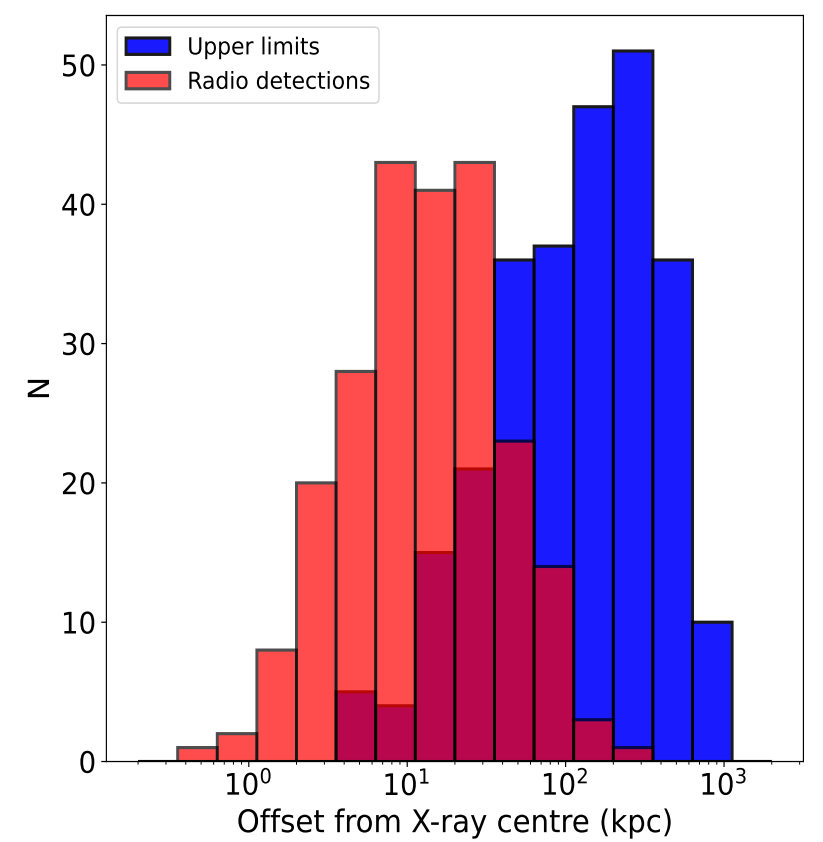

Fig. 5. Histogram showing BCG offsets from the X-ray emission peak, assumed as the centre of the cluster/group, for galaxies with AGN radio emission (red) and for those with radio upper limits (blue).

have also been discussed in Burns (1990); Best et al. (2007); Cavagnolo et al. (2008); Shen et al. (2017). On the other hand, off-centre galaxies have to rely on more episodic processes, such as cluster/group mergers and/or galaxy interactions. We find the same results in this sample since, as discussed above, radio-loud AGNs are mostly found at offset $<50 \mathrm{kpc}$.

\subsection{The extent of $B C G$ radio galaxies}

Radio galaxies exhibit a plethora of different shapes and sizes. The reasons for the unusual size of some giant radio galaxies (e.g., Brüggen et al. 2021; Dabhade et al. 2020) and for the significantly smaller extent of some others (e.g. FR0, Baldi et al. 2015) have been investigated previously. Hardcastle et al. (2019) presented the largest up-to-date sample of radio galaxies in which the relation between the radio power and the linear size was investigated. The location of a source in this diagram is indicative of its initial conditions and current evolutionary state (Hardcastle 2018). Kolokythas et al. (2018) found a clear link between the $235 \mathrm{MHz}$ power and the projected Largest Linear Size (LLS) of their resolved radio galaxies. The same relation was already found for cluster and field radio galaxies by Ledlow et al. (2002), and is investigated for our sample of BCGs at 144 $\mathrm{MHz}$ (top panel of Fig. 6). The LLS of radio galaxies was manually measured from the LOFAR eFEDS mosaic, assuming error equal to the synthesised beam. To exclude unresolved sources, only those with Largest Angular Size LAS $>2 \times$ beam are taken into account.

Most sources show LLS between 100 and $300 \mathrm{kpc}$, with the mean at LLS $\sim 235 \mathrm{kpc}$ and standard deviation $\sim 160 \mathrm{kpc}$. Large sources mostly show a classical double-lobed morphology, while the smallest ones are point-like. As previously observed, there is a positive correlation between LLS and luminosity, with larger radio galaxies being more powerful. We see that the relation holds even at relatively high luminosities ${ }^{5}$. Nevertheless, we note that we are likely missing large, low-power radio sources be-

${ }^{5}$ CLoGS only reaches $L_{R} \sim 10^{25} \mathrm{~W} \mathrm{~Hz}^{-1}$ at $235 \mathrm{MHz}$. 

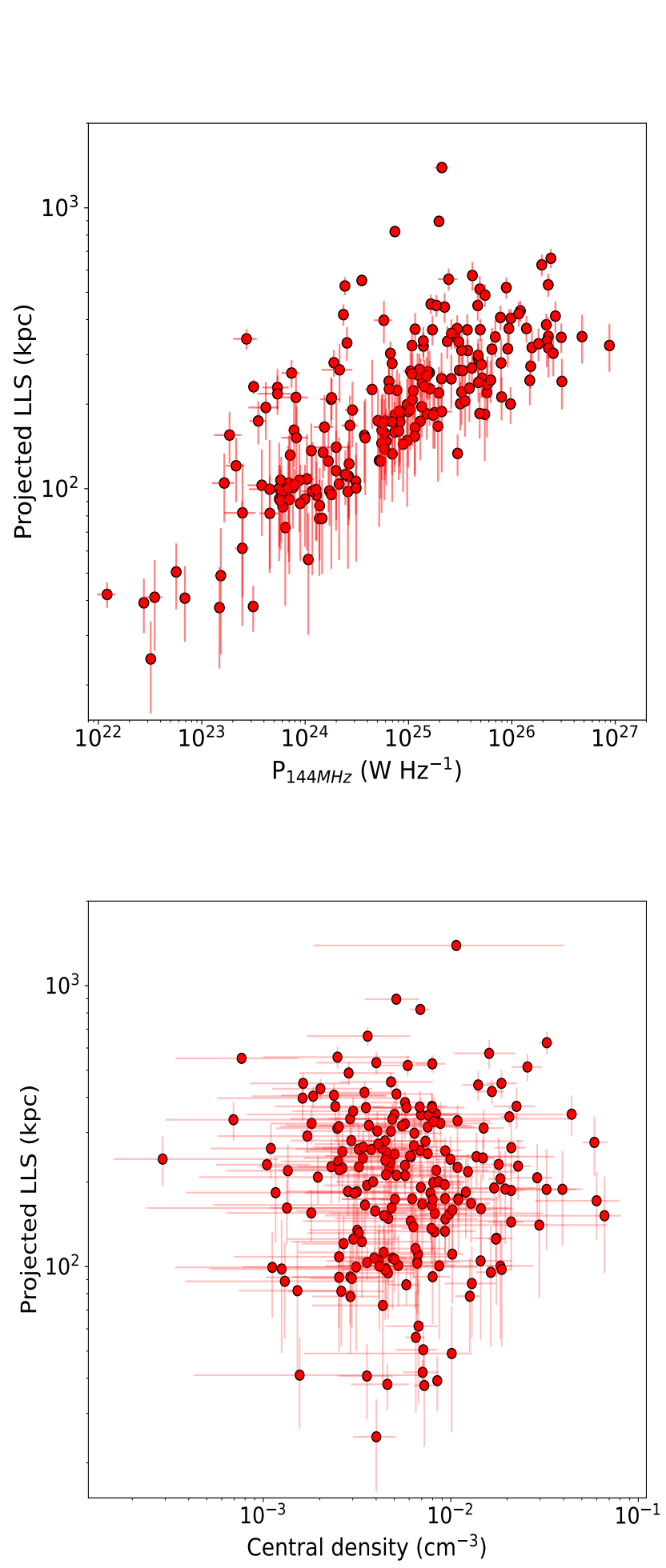

Fig. 6. Top: Projected Largest Linear Size (LLS) vs. radio power of eFEDS radio galaxies. Bottom: Projected LLS of the radio galaxy vs. ICM central density.

cause of surface brightness limitations. This issue has been extensively addressed in Hardcastle et al. (2019) making use of a significantly larger sample (23344 objects) of radio galaxies.

Multiple environmental factors are likely to contribute to the size of the radio source. The most important one is the age, which necessarily introduces scatter into any relation with other physical quantities. Other factors include the location of the galaxy within the host cluster, the density of the ICM at the posi-

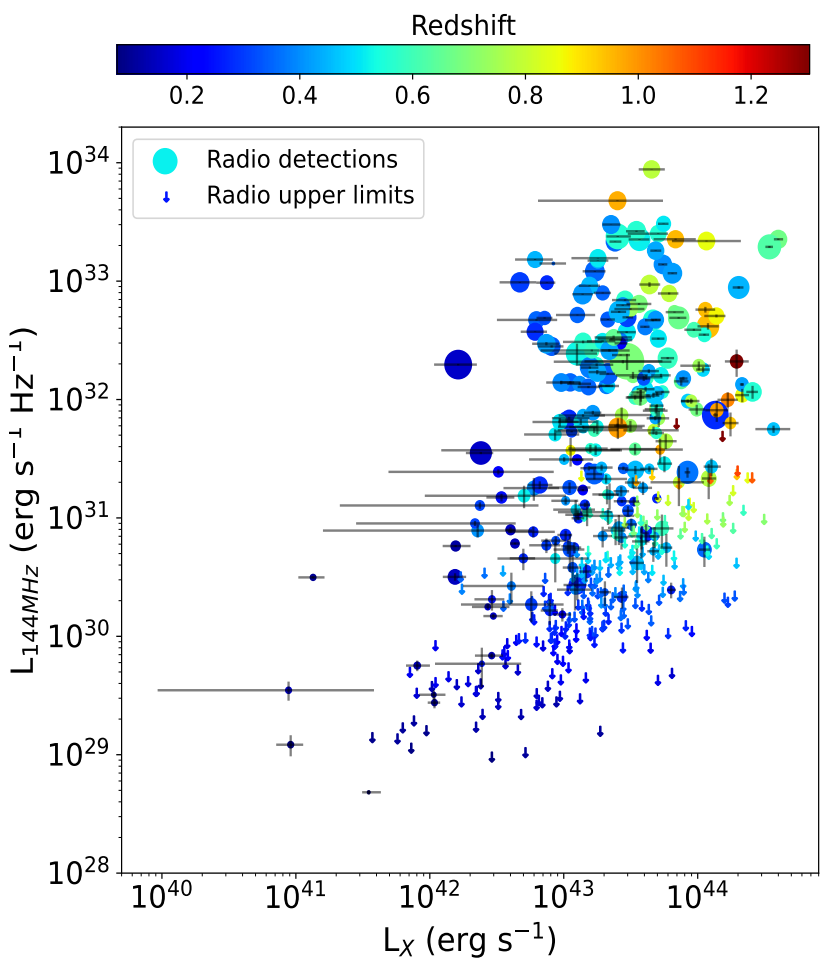

Fig. 7. $144 \mathrm{MHz}$ power of radio galaxies vs. X-ray luminosity of the host cluster. Symbol sizes are proportional to the LLS of the source and their color indicates the redshift. Downward pointing arrows denote radio upper limits. Bars represent errors on both axes.

tion of the galaxy, the efficiency of the accretion onto the AGN, the radio power of the outburst and others (see e.g. Moravec et al. 2020, and references therein).

To this end, in the bottom panel of Fig. 6 we show the LLS of the radio galaxy plotted against the central density (at $R=0.02 R_{500}$ ) of the host cluster, obtained by fitting the cluster model by Vikhlinin et al. (2006) to density profiles (see G21 for further details). We see no correlation of the LLS with the central density, suggesting that radio power is more prominent than ambient density in determining the size of the radio galaxy and that the contribution of other factors could affect a possible link.

\subsection{Correlation between $\mathrm{X}$-ray and $144 \mathrm{MHz}$ radio luminosity}

In P20, we have studied the correlation between the $1.4 \mathrm{GHz}$ power of radio galaxies and the X-ray luminosity of the host group for 247 galaxy groups in COSMOS. A similar correlation between the mass of galaxy clusters, known to correlate with the X-ray luminosity (e.g., Lovisari et al. 2020), and the radio power of BCGs has been found by Hogan et al. (2015). Here, we focus on the same relation, albeit at the lower radio frequency of 144 $\mathrm{MHz}$.

Fig. 7 shows the $144 \mathrm{MHz}$ power of the radio galaxy plotted against the X-ray luminosity of the host group/cluster. The size of the symbols is proportional to the LLS of the radio sources, and the colour corresponds to the redshift. Upper limits are represented by downward pointing arrows. There is a clear trend for stronger radio galaxies to be hosted in more X-ray luminous clusters, as found by P20. However, the significant number of radio upper limits makes it harder to determine whether the observed correlation is real or produced by selection effects set by the sensitivity of the observation. 


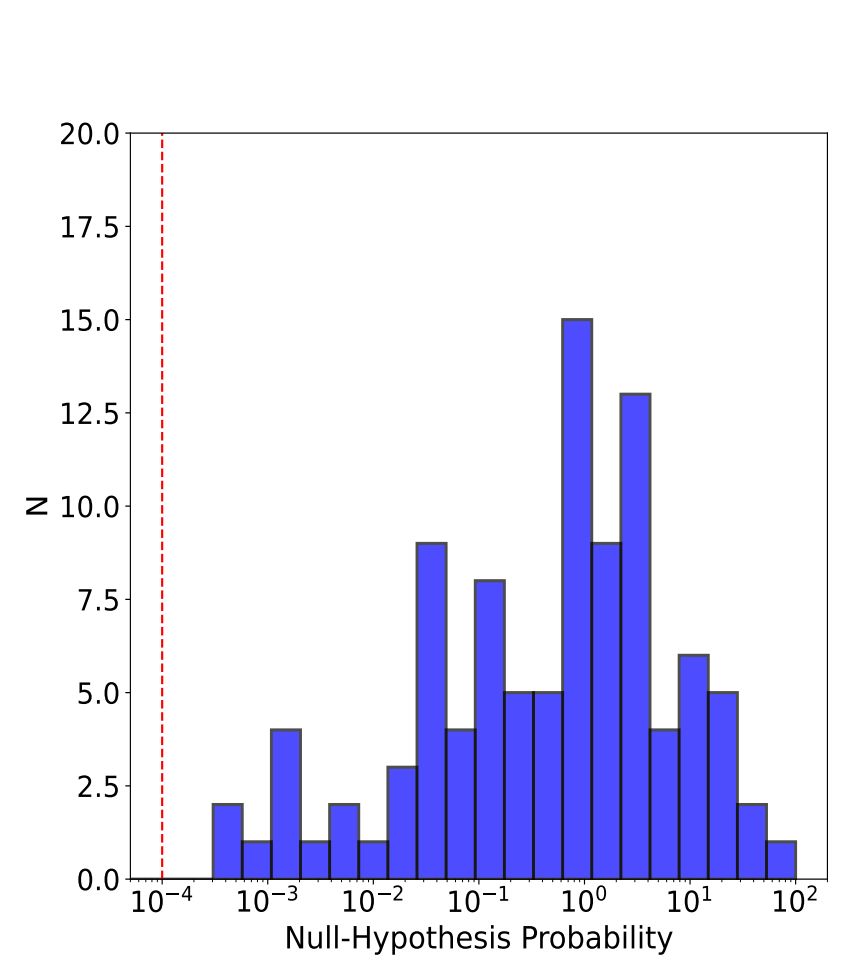

Fig. 8. The histogram shows the null-hypothesis probability distribution that the correlation is not real for 100 mock datasets produced by the scrambling test. The red dashed line indicates the probability of the real sample.

To ascertain if the correlation is genuinely detected, we performed the partial correlation Kendall's $\tau$ (Akritas \& Siebert 1996) test. This tool has already been used in a number of papers (e.g., Ineson et al. 2015; Pasini et al. 2020) to test correlations in the presence of upper limits and redshift-dependence. The algorithm estimates the null-hypothesis probability that selection effects are producing the correlation. If the probability is low, then it is likely that the correlation is real. The test performed on our sample gives a null-hypothesis probability $p<0.0001 \%$ ( $\tau=0.1178, \sigma=0.0227$ ), indicating that the correlation is real and not generated by selection effects. This result is consistent with P20, who also found that such a correlation, but at higher frequency, was not produced by biases.

Bianchi et al. (2009) argued that the Kendall's $\tau$ test may underestimate the redshift contribution, particularly when it comes to determining the significance and the functional relation. For this reason, they performed a 'scrambling' test that has also been used in other works (e.g., Bregman 2005; Merloni et al. 2006). The principle of this algorithm is to keep each $L_{X} / z$ pair since their association comes from the source selection. Then they shuffle the corresponding radio fluxes, assigning them to a new $L_{X} / z$ pair. The new radio luminosity is then computed at the new redshift (see Eq. 2). If the correlation is real, one expects that it disappears when shuffling the luminosity pairs. We applied this test 100 times and for each cycle estimated the nullhypothesis probability through the Kendall $\tau$ test. Results are shown in Fig. 8. Out of 100 cycles, the null-hypothesis probability is never found to be lower than the real sample. The mean probability value lies at $\sim 4 \%$, with a standard deviation of $\sim 9 \%$, while the peak lies between $0.7 \%$ and $5 \%$. This result supports the hypothesis that the observed correlation is real.

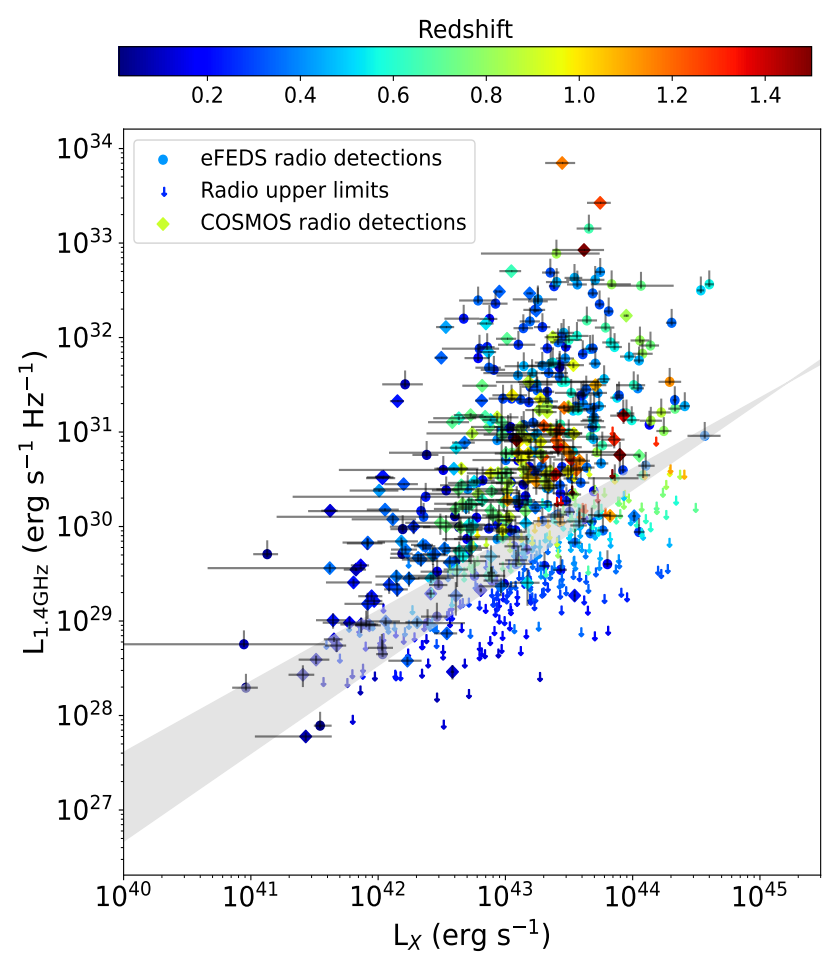

Fig. 9. 1.4 GHz power of radio galaxies vs. X-ray luminosity of the host cluster for both eFEDS and P20 samples. The colours correspond to the redshift. eFEDS data is represented by circles, while diamonds are COSMOS systems. Downward pointing arrows denote radio upper limits. Bars represent errors in both axes. Errors on $y$-axis are dominated by the assumed uncertainty on the spectral index. The best-fit relation is shown in grey: $\log L_{R}=(0.84 \pm 0.09) \log L_{X}-(6.46 \pm 4.07)$.

\subsection{The $X$-ray/radio correlation at $1.4 \mathrm{GHz}$}

We compare our $144 \mathrm{MHz}$ sample in eFEDS with a subsample of 137 systems among the 247 COSMOS galaxy groups studied at $1.4 \mathrm{GHz}$ in P20. A further cross-match of our sample with all-sky surveys at this frequency (e.g. NVSS, Condon et al. 1998) is not trivial, due to significant differences in surface brightness sensitivity and resolution. For this reason, the $144 \mathrm{MHz}$ luminosities were converted to luminosities at a frequency of $1.4 \mathrm{GHz}$ assuming $\alpha=0.8 \pm 0.2$. The assumed uncertainty on the spectral index dominates on the previous $144 \mathrm{MHz}$ flux error. Combining the two catalogs, we get a total of 364 galaxy clusters and groups that allow us to assess the radio/X-ray correlation using a larger sample. The corresponding $\log L_{R}-\log L_{X}$ plot is shown in Fig. 9.

The distributions of COSMOS and eFEDS clusters and groups are in good agreement. This is confirmed by the twodimensional Kolmogorov-Smirnov test that, under the nullhypothesis that the two samples are drawn from the same parent distribution, gives $p=0.41$. This implies that our assumption of a uniform spectral index of $\alpha=0.8$ for every radio galaxy is valid, although it introduces more scatter in the correlation. Still, a clear trend for more massive groups and clusters hosting more powerful radio sources is seen. This is also supported by the Kendall's $\tau$ test, that for eFEDS+COSMOS gives $p<0.0001$ ( $\tau=0.1331, \sigma=0.0138)$. The best-fit relation was estimated exploiting the parametric EM algorithm coded in the AStronomical SURVival statistics package (ASURV, Feigelson et al. 2014), that takes into account different contributions by detections and upper limits. We find $\log L_{R}=(0.84 \pm 0.09) \log L_{X}-(6.46 \pm 4.07)$. 


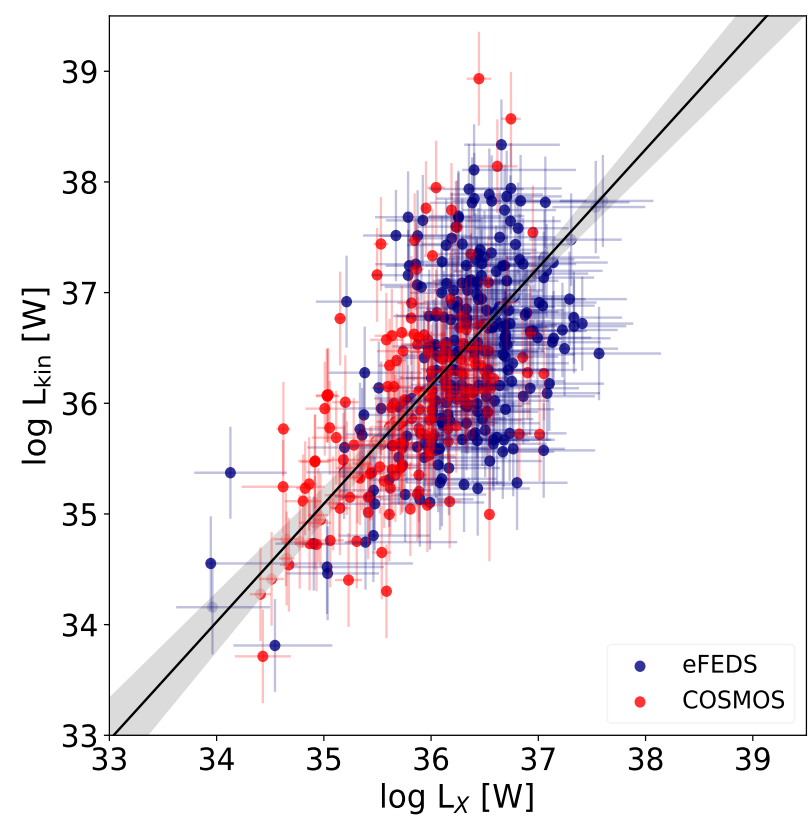

Fig. 10. Kinetic luminosity of BCG radio galaxies estimated at $1.4 \mathrm{GHz}$ vs. X-ray luminosity of the host cluster/group for the eFEDS (blue) and P20 (red) samples. The black line represents the best-fit estimated from Bayesian inference: $\log L_{\text {kin }}=(1.07 \pm 0.11) \log L_{X}-(2.19 \pm 4.05)$. The grey area indicates $1 \sigma$ errors.

This estimate is marginally consistent with the best-fit relations of P20 $\left(\log L_{R}=(1.07 \pm 0.12) \times \log L_{X}-(15.90 \pm 5.13)\right.$ and Pasini et al. (2021a) $\left(\log L_{R}=(0.94 \pm 0.43) \times \log L_{X}-(9.53 \pm 18.19)\right)$, obtained through the same method and applying Bayesian inference, respectively.

The correlation may imply a link between radiative cooling from the ICM, and the more variable and episodic activity of the AGN. Since the X-ray luminosity is predominantly driven by the cluster or group mass, such a correlation may be produced by massive clusters hosting more massive BCGs, and in turn more massive BHs. In relaxed clusters, the cooling of the ICM is able to efficiently feed the central AGN, leading to higher radio powers (Soker \& Pizzolato 2005; Gaspari et al. 2011). This is reflected in the well-studied link between the cavity power of systems hosting X-ray bubbles and the luminosity of the cluster cooling region (e.g., Bîrzan et al. 2004; Rafferty et al. 2006; Bîrzan et al. 2017). Sun (2009) also argued that small coronae of X-ray emitting gas in BCGs are able to trigger strong radio outbursts long before cool cores are formed in the host cluster, leading to heating in their surroundings and even preventing their formation, especially in low mass systems. The correlation presented here shows a large scatter, especially at high luminosities. This could be caused, e.g. by differences in the dynamical states, which we will explore in the next section.

\subsection{Kinetic luminosity and AGN feedback}

The radio luminosity is a measure of the instantaneous radiative loss rate of the radio lobes, and as such is only indirectly related to the energy produced by the AGN through accretion onto the SMBH. For an active source, only a small fraction of the total power supplied to the lobes has been radiated away at any given time, while a much larger fraction is stored in the lobes and a similar amount has been dissipated into the surrounding ICM during the expansion of the jets through the ICM (Willott et al. 1999; Smolčić et al. 2017). The latter, which we will refer to as kinetic luminosity, is directly linked to the heating of the ICM and contributes to quench the radiative losses of the hot plasma (see Sec. 1 for references).

The relation between the kinetic and radio luminosity has been the subject of ample work (e.g., Willott et al. 1999; Bîrzan et al. 2004, 2008; Cavagnolo et al. 2010; O’Sullivan et al. 2011b; Smolčić et al. 2017). As thoroughly discussed in Hardcastle et al. (2019), there are currently two methods to infer the kinetic luminosity. The first one relies on the identification of X-ray cavities and is affected by assumptions on the cavity age and biased towards small sources in cluster rich environments (Bîrzan et al. 2012). The second method relies on a conversion based on a theoretical model and, as such, can lead to unrealistic results if the contribution of source age, environment and redshift to the radio luminosity are not taken into account properly. We refer to Hardcastle et al. (2019) and Appendix A of Smolčić et al. (2017) for a detailed discussion of this scaling relation. Here, we assume the relation adopted by Willott et al. (1999) for converting to the 1.4 $\mathrm{GHz}$ rest-frame luminosity (Heckman \& Best 2014):

$\log L_{\mathrm{kin}, 1.4 \mathrm{GHz}}=0.86 \log L_{1.4 \mathrm{GHz}}+14.08+1.5 \log f_{W}$,

where $L_{\mathrm{kin}, 1.4 \mathrm{GHz}}$ is the kinetic luminosity, $L_{1.4 \mathrm{GHz}}$ is the luminosity as measured at $1.4 \mathrm{GHz}$, while $f_{W}$ is an uncertainty parameter that we assume $f_{W}=15$, as estimated by X-ray observations of ICM bubbles in galaxy clusters (e.g., Merloni \& Heinz 2007; Bîrzan et al. 2008). We determine the kinetic luminosity for the radio galaxies of the eFEDS and the P20 sample, and we compare it to the X-ray luminosity within $500 \mathrm{kpc}$ of the host cluster. The result is shown in Fig. 10.

In order to infer the relationship between the X-ray and the kinetic luminosity, we applied Bayesian inference on the two samples using the linmix ${ }^{6}$ package (Kelly 2007). With this tool, we performed a linear fit in the log-log scale in the form:

$Y=\alpha+\beta X+\epsilon$,

with $\alpha$ and $\beta$ representing the intercept and the slope, respectively, while $\epsilon$ is the intrinsic scatter of the relation. We find $\alpha=-2.19 \pm 4.05, \beta=1.07 \pm 0.11$ and $\epsilon=0.25 \pm 0.05$. We notice that the conversion from radio to kinetic luminosity, which also depends on external factors - such as the morphology and age of the radio source, the extrapolation of $1.4 \mathrm{GHz}$ fluxes, or the surrounding environment - and relies on theoretical models, may have introduced artificial scatter into the correlation.

Nevertheless, the plot suggests that in most clusters and groups the heating from the central AGN efficiently counterbalances the ICM radiative losses, as already found in a large number of publications (see references above and McNamara \& Nulsen 2007, 2012 for reviews). However, most of these papers take into account the luminosity from within the cooling region, which is usually defined as the cluster region within which the cooling time of the ICM is shorter than $7.7 \mathrm{Gyr}$. These usually range between $\sim 50$ and $\sim 150 \mathrm{kpc}$ (Bîrzan et al. 2017), and their extent can only be estimated through deprojected analysis of the thermodynamical profiles (i.e. temperature, density, cooling time) derived from X-ray observations. The detection of cavities

6 https://github.com/jmeyers314/linmix 

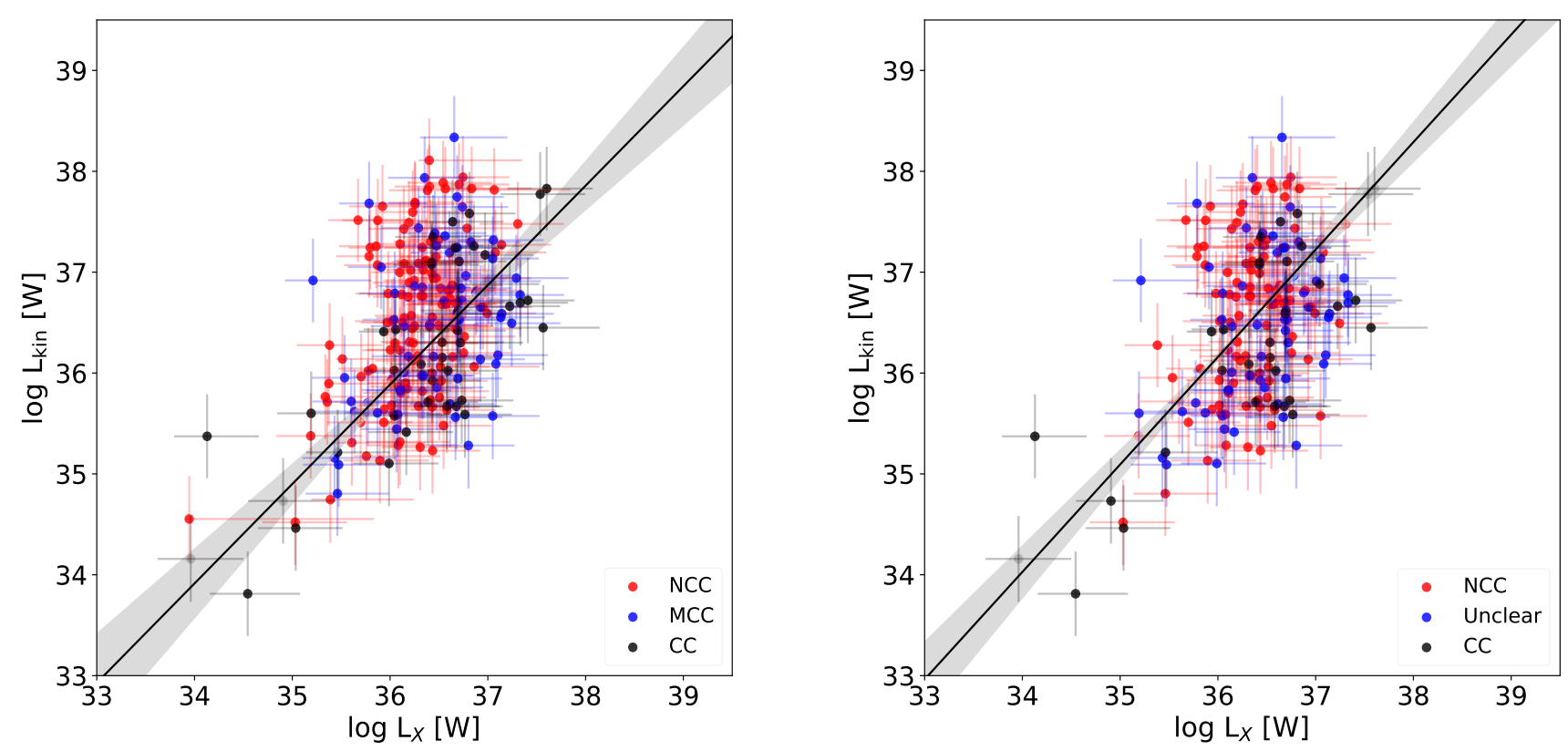

Fig. 11. Left: Kinetic luminosity of BCG radio galaxies estimated at $1.4 \mathrm{GHz}$ vs. X-ray luminosity of the host cluster/group for the eFEDS sample. Data are classified into non-cool cores (red), moderate cool cores (blue) and cool cores (black) based on the concentration parameter. Right: Same of left Panel, with data classified based on the $R_{\text {score }}$ (see discussion).

as a tell-tale for AGN heating (McNamara et al. 2000; Bîrzan et al. 2004) usually requires deep, high-resolution X-ray observations as well.

The kinetic luminosity-X-ray luminosity relation, estimated through survey data - albeit with the unprecedented sensitivity of eROSITA - is able to provide a first insight into the processes of AGN feedback of a large number of clusters and groups. Kinetic and X-ray luminosity act as proxy for mechanical feedback and cooling luminosity, respectively, which together constitute the 'parent' correlation usually found in cool core clusters. Nevertheless, here the analysis is performed on all our objects, with no distinction between cool cores and merging clusters. Main et al. (2017) found that, in their sample of clusters, such a correlation only holds for cool cores. Their classification is based on the central cooling time, determined through Chandra observations at $0.004 R_{500}$ by Hudson et al. (2010). The eROSITA observations do not yield cooling times at such small cluster-centric radii and we are not able to reproduce the same classification for our objects. Instead we quantify the dynamical state of clusters via the concentration parameter as defined in Lovisari et al. (2017) and estimated for eFEDS clusters in G21 as:

$$
c_{\mathrm{SB}}=\frac{S_{B}\left(<0.1 R_{500}\right)}{S_{B}\left(<R_{500}\right)},
$$

where $S_{B}$ is the surface brightness estimated inside $0.1 R_{500}$ for the numerator, and inside $R_{500}$ for the denominator. This parameter is an indicator of the presence of a centrally peaked X-ray surface brightness profile, which correlates with the dynamical state of the cluster. Lovisari et al. (2017) discusses the use of different thresholds to classify clusters into cool cores and disturbed systems, showing how completeness (i.e. being able to pick all clusters belonging to a given class) and purity (i.e. being able to securely assign clusters to a given class) change depending on the chosen threshold. Here, following the work cited above, we choose to define as non-cool cores (NCC) clusters with $c_{\mathrm{SB}}<0.15$, while cool cores (CC) have $c_{\mathrm{SB}}>0.27$. This classification allows for $100 \%$ purity for both subsamples, albeit completeness goes down to $\sim 53 \%$ for $\mathrm{CC}$ and $\sim 75 \%$ for NCC (see Lovisari et al. 2017 for more details). Clusters with $0.15<c_{\mathrm{SB}}<0.27$ cannot be securely categorised, and will be arbitrarily referred to as moderate cool cores (MCC). In the left panel of Fig. 11 we show the $L_{\text {kin }}-L_{X}$ plot for the eFEDS cluster sample, in which clusters were classified via the concentration parameter. We find that $\sim 53 \%$ of clusters are NCC, $\sim 28 \%$ are MCC and $\sim 19 \%$ are CC. We see no obvious difference in the distribution between the three subsamples. Therefore, the dynamical state of the cluster does not seem to have a large effect on the scatter in the $\mathrm{X}$-ray/radio relation.

As discussed in more details in G21, the concentration acts as an indicator of the presence of a cool core. However, while a relaxed cluster will generally present a cool core, a cool core is not always an indication of relaxation: a merger in its initial stage affects predominantly the cluster outskirts and does not disrupt the cool core (see e.g. theoretical work by Rasia et al. 2015 and Biffi et al. 2016). Therefore, classifying the dynamical state of clusters based on concentration alone is useful to distinguish disturbed objects with low concentration, but does not provide a clear identification of relaxed clusters (see Fig. 9 of G21 and related discussion). For this reason, we perform an alternative classification based on a new morphological parameter first introduced in the same paper, the so-called Relaxation score $\left(R_{\text {score }}\right)$. Since a complete, physical definition of this parameter requires detailed discussions about a number of parameters (see below), we remind to G21 for more insights, and here we just provide a brief description. The $R_{\text {score }}$ combines a number of morphological parameters usually determined for galaxy clusters, such as concentration, central density, ellipticity (ratio between minor and major axes of the cluster), cuspiness (slope 
of the density profile at a given radius) and others. The resulting $R_{\text {score }}$ provides a more clear indication, with respect to concentration alone, of the dynamical state of a cluster. In particular, the $R_{\text {score }}$ should be higher for relaxed objects, that show large concentration, central density, ellipticity and cuspiness. On the other hand, the same parameter should decrease in disturbed clusters.

Following the discussion in G21, we define as relaxed objects with $R_{\text {score }}>0.0137$. The results of this alternative classification are shown in the right panel of Fig. 11. We only plot clusters for which a proper estimate of the $R_{\text {score }}$ was feasible in G21. Objects classified as relaxed through the $R_{\text {score }}$ and as $\mathrm{CC}$ through the concentration are generally referred to as $\mathrm{CC}$, those with low $R_{\text {score }}$ and concentration are NCC, while clusters with high concentration (same threshold used for left panel) but $R_{\text {score }}<0.0137$ are labeled as unclear. We remind to G21 for discussions and comparisons between different classifications, while in this work we focus on the correlation.

Even when introducing a more accurate parameter such as the $R_{\text {score }}$, there is still not a clear distinction in the distribution between cool cores and merging objects, as instead found e.g. in Main et al. (2017). Furthermore, it is not clear how such a relation could be present in disturbed systems. In these objects, the cooling of the ICM is slow and BCGs often hard to identify. Morphological parameters have been widely used to determine the dynamical state of clusters, but a more secure classification based on central cooling time may be more useful to understand in which clusters a connection of AGN and cooling ICM can ensue. A possibility is that the link between AGN and their environment could be produced, even in disturbed objects, by rapidly-cooling coronae permeating the host galaxy (Sun et al. 2007; Gastaldello et al. 2008; Sun 2009). This idea has been already suggested for NCC hosting radio AGN, such as A2028 (Gastaldello et al. 2010). It is also plausible that small, lowentropy regions of the cluster core such as cool core remnants (Rossetti \& Molendi 2010) could affect the AGN, leading to the observed relation. Another possibility is that NCC do not belong, in fact, to the correlation. To test this, we checked the scatter of the correlation after applying Bayesian inference only on CC. If NCC are not part of the correlation, the scatter of the data should decrease when fitting only CC. We find $\epsilon=0.17 \pm 0.10$, consistent within errors with the previous estimate. Nevertheless, the uncertainty increases because of the relatively small number of $\mathrm{CC}$, and further analyses exploiting larger samples are needed to investigate this further.

\section{Conclusions}

We make use of eROSITA (X-ray) and LOFAR (radio) observations of the eFEDS field in order to investigate radio galaxies hosted in BCGs. Our results can be summarised as follows:

- Our sample yields 227 detections and 248 upper limits in the redshift range $0.01<z<1.3$ and luminosity range $10^{22}-$ $10^{27} \mathrm{~W} \mathrm{~Hz}^{-1}$ at $144 \mathrm{MHz}$. The remaining 67 clusters were excluded from the analysis to avoid contamination by missclassified AGN (see Sec. 2.3). The radio detection rate is $\sim 48 \%$, which is lower than in other samples of well-studied groups and clusters.

- BCGs hosting radio-loud AGN mostly ( 84\%) lie within 50 $\mathrm{kpc}$ from the cluster centre. BCGs that are more offset tend to have lower levels of radio emission, or lie below our detection threshold.

- As already argued in previous works, larger radio galaxies are usually more powerful. However, we note that a relevant selection effect is present in our sample, since we lack large, low-power radio sources because of surface brightness limitations. We see no correlation of the cluster's central ( $\left.R=0.02 R_{500}\right)$ density with the LLS, suggesting that the luminosity is a better predictor for the size of the radio galaxy.

- We studied the relation between the $144 \mathrm{MHz}$ radio galaxy power and the host cluster X-ray luminosity measured within $500 \mathrm{kpc}$ from the cluster centre, finding a positive correlation. Because of the large number of upper limits, we relied on statistical tests, such as the partial correlation Kendall's $\tau$ test and the scrambling test, to show that the correlation is not produced by selection effects in the radio band.

- Converting the $144 \mathrm{MHz}$ power of radio galaxies to $1.4 \mathrm{GHz}$, we compared our results with the correlation between the $\mathrm{X}$ ray luminosity and the $1.4 \mathrm{GHz}$ power of a COSMOS galaxy groups sample first investigated by Pasini et al. (2020). We found that the two samples are in good agreement based on a Kolmogorov-Smirnov test that, under the null-hypothesis that the samples are drawn from the same parent distribution, gives $p=0.41$. We estimated a best-fit relation $\log L_{R}=$ $(0.84 \pm 0.09) \log L_{X}-(6.46 \pm 4.07)$.

- We converted the radio powers of radio galaxies to kinetic luminosities, making use of widely used scaling relations. Comparing the kinetic luminosity to the X-ray luminosity within $500 \mathrm{kpc}$ from the cluster centre, we found that in most objects the ICM's radiative losses are efficiently counterbalanced by heating supplied from the central AGN. We derived the best-fit relation applying Bayesian inference, obtaining $\log L_{\text {kin }}=(-2.19 \pm 4.05)+(1.07 \pm 0.11) \log L_{X}+(0.25 \pm 0.05)$.

- We classified eFEDS clusters into disturbed and relaxed objects based on two different parameters, concentration and Relaxation score (see Sec. 3.6 for a definition). We could see no significant differences in the $L_{\text {kin }}-L_{X}$ relation between the subsamples.

Future prescriptions of radio-mode AGN feedback in simulations need to be able to recover the properties described in this paper. In addition to massive halo gas fractions, entropy slopes, and galaxy properties, they need to recover radio luminosities as a function of the host cluster properties. With the new all-sky Xray surveys, a correlation between the cluster X-ray luminosity and the BCG radio power can be used to probe AGN feedback across a larger range of host masses and to control for the effect of other observables. Particularly, the synergy between eRASS (Bulbul et al. in prep.) and the LOFAR Two-Metre Sky Survey (LoTSS, Shimwell et al. 2017), as well as the forthcoming LOFAR LBA Sky Survey (LoLSS, de Gasperin et al. 2021), will provide samples of thousands of clusters and groups for which the interplay between the AGN and the ICM can be investigated.

\section{Acknowledgements}

TP thanks Philip Best for useful comments. TP is supported by the BMBF Verbundforschung under grant number 50OR1906. MB acknowledges support from the Deutsche Forschungsgemeinschaft under Germany's Excellence Strategy - EXC 2121 "Quantum Universe" - 390833306. DNH acknowledges support from the ERC through the grant ERC-Stg DRANOEL n. 714245. $\mathrm{AB}$ acknowledges support from the VIDI research programme with project number 639.042.729, which is financed by the Netherlands Organisation for Scientific Research (NWO). FG acknowledges support from INAF mainstream project 'Galaxy Clusters Science with LOFAR' 1.05.01.86.05. RJvW acknowledges support from the ERC Starting Grant ClusterWeb 804208. WLW acknowledges support from the CAS-NWO programme 
for radio astronomy with project number 629.001.024, which is financed by the Netherlands Organisation for Scientific Research (NWO). This work is based on data from eROSITA, the soft X-rays instrument aboard SRG, a joint Russian-German science mission supported by the Russian Space Agency (Roskosmos), in the interests of the Russian Academy of Sciences represented by its Space Research Institute (IKI), and the Deutsches Zentrum für Luft- und Raumfahrt (DLR). The SRG spacecraft was built by Lavochkin Association (NPOL) and its subcontractors, and is operated by NPOL with support from the Max Planck Institute for Extraterrestrial Physics (MPE). The development and construction of the eROSITA X-ray instrument was led by MPE, with contributions from the Dr. Karl Remeis Observatory Bamberg \& ECAP (FAU Erlangen-Nuernberg), the University of Hamburg Observatory, the Leibniz Institute for Astrophysics Potsdam (AIP), and the Institute for Astronomy and Astrophysics of the University of Tübingen, with the support of DLR and the Max Planck Society. The Argelander Institute for Astronomy of the University of Bonn and the Ludwig Maximilians Universität Munich also participated in the science preparation for eROSITA. The eROSITA data shown here were processed using the eSASS/NRTA software system developed by the German eROSITA consortium. LOFAR data products were provided by the LOFAR Surveys Key Science project (LSKSP; https://lofar-surveys.org/) and were derived from observations with the International LOFAR Telescope (ILT). LOFAR (van Haarlem et al. 2013) is the Low Frequency Array designed and constructed by ASTRON. It has observing, data processing, and data storage facilities in several countries, that are owned by various parties (each with their own funding sources), and that are collectively operated by the ILT foundation under a joint scientific policy. The efforts of the LSKSP have benefited from funding from the European Research Council, NOVA, NWO, CNRSINSU, the SURF Co-operative, the UK Science and Technology Funding Council and the Jülich supercomputing centre.

\section{References}

Aihara, H., Arimoto, N., Armstrong, R., et al. 2018, PASJ, 70, S4 Akritas, M. G. \& Siebert, J. 1996, MNRAS, 278, 919

Alexander, D. M., Swinbank, A. M., Smail, I., McDermid, R., \& Nesvadba, N. P. H. 2010, MNRAS, 402, 2211

Baldi, R. D., Capetti, A., \& Giovannini, G. 2015, A\&A, 576, A38

Best, P. N., Kauffmann, G., Heckman, T. M., et al. 2005, MNRAS, 362, 25

Best, P. N., von der Linden, A., Kauffmann, G., Heckman, T. M., \& Kaiser, C. R. 2007, MNRAS, 379, 894

Bharadwaj, V., Reiprich, T. H., Lovisari, L., \& Eckmiller, H. J. 2015, 573, A75

Bharadwaj, V., Reiprich, T. H., Schellenberger, G., et al. 2014, 572, A46

Bianchi, S., Bonilla, N. F., Guainazzi, M., Matt, G., \& Ponti, G. 2009, A\&A, 501,915

Biffi, V., Borgani, S., Murante, G., et al. 2016, ApJ, 827, 112

Bîrzan, L., McNamara, B. R., Nulsen, P. E. J., Carilli, C. L., \& Wise, M. W. 2008, ApJ, 686, 859

Bîrzan, L., Rafferty, D. A., Brüggen, M., \& Intema, H. T. 2017, MNRAS, 471, 1766

Bîrzan, L., Rafferty, D. A., McNamara, B. R., Wise, M. W., \& Nulsen, P. E. J. 2004, ApJ, 607, 800

Bîrzan, L., Rafferty, D. A., Nulsen, P. E. J., et al. 2012, MNRAS, 427, 3468

Blanton, M. R., Bershady, M. A., Abolfathi, B., et al. 2017, AJ, 154, 28

Böhringer, H., Chon, G., \& Collins, C. A. 2014, A\&A, 570, A31

Botteon, A., Brunetti, G., van Weeren, R. J., et al. 2020, ApJ, 897, 93

Bregman, J. N. 2005, arXiv e-prints, astro

Brüggen, M., Reiprich, T. H., Bulbul, E., et al. 2021, A\&A, 647, A3

Brunner, H., Liu, T., Lamer, G., et al. 2021, A\&A, (submitted)

Burns, J. O. 1990, AJ, 99, 14

Cavagnolo, K. W., Donahue, M., Voit, G. M., \& Sun, M. 2008, ApJ, 683, L107

Cavagnolo, K. W., Donahue, M., Voit, G. M., \& Sun, M. 2009, ApJS, 182, 12

Cavagnolo, K. W., McNamara, B. R., Nulsen, P. E. J., et al. 2010, ApJ, 720, 1066

Cavaliere, A. \& Fusco-Femiano, R. 1976, A\&A, 49, 137

Condon, J. J., Cotton, W. D., Greisen, E. W., et al. 1998, AJ, 115, 1693
Croton, D. J., Springel, V., White, S. D. M., et al. 2006, MNRAS, 365, 11 Dabhade, P., Röttgering, H. J. A., Bagchi, J., et al. 2020, A\&A, 635, A5 de Gasperin, F., Dijkema, T. J., Drabent, A., et al. 2019, A\&A, 622, A5 de Gasperin, F., Williams, W. L., Best, P., et al. 2021, A\&A, 648, A104 De Propris, R., West, M. J., Andrade-Santos, F., et al. 2021, MNRAS, 500, 310 Dey, A., Schlegel, D. J., Lang, D., et al. 2019, AJ, 157, 168

Driver, S. P., Norberg, P., Baldry, I. K., et al. 2009, Astronomy and Geophysics, $50,5.12$

Dunn, R. J. H., Allen, S. W., Taylor, G. B., et al. 2010, MNRAS, 404, 180 Ebeling, H., Edge, A. C., Fabian, A. C., et al. 1997, ApJ, 479, L101

Ehlert, S., Allen, S. W., von der Linden, A., et al. 2011, MNRAS, 411, 1641

Fabian, A. C., Sanders, J. S., Taylor, G. B., et al. 2006, MNRAS, 366, 417

Feigelson, E. D., Nelson, P. I., Isobe, T., \& LaValley, M. 2014, ASURV: Astronomical SURVival Statistics

Gaspari, M., Melioli, C., Brighenti, F., \& D’Ercole, A. 2011, MNRAS, 411, 349 Gaspari, M., Tombesi, F., \& Cappi, M. 2020, Nature Astronomy, 4, 10

Gastaldello, F., Buote, D. A., Brighenti, F., \& Mathews, W. G. 2008, ApJ, 673, L17

Gastaldello, F., Buote, D. A., Temi, P., et al. 2009, ApJ, 693, 43

Gastaldello, F., Ettori, S., Balestra, I., et al. 2010, A\&A, 522, A34+

Ghirardini, V., Bahar, Y. E., Bulbul, E., et al. 2021, A\&A, (submitted)

Giacintucci, S., O’Sullivan, E., Vrtilek, J., et al. 2011, ApJ, 732, 95

Giodini, S., Smolčić, V., Finoguenov, A., et al. 2010, ApJ, 714, 218

Gitti, M., Brighenti, F., \& McNamara, B. R. 2012, Advances in Astronomy, 2012 [arXiv: 1109.3334]

Gitti, M., O’Sullivan, E., Giacintucci, S., et al. 2010, ApJ, 714, 758

Hamer, S. L., Edge, A. C., Swinbank, A. M., et al. 2016, MNRAS, 460, 1758

Hardcastle, M. J. 2018, ArXiv e-prints [arXiv: 1801.00667]

Hardcastle, M. J., Shimwell, T. W., Tasse, C., et al. 2021, A\&A, 648, A10

Hardcastle, M. J., Williams, W. L., Best, P. N., et al. 2019, A\&A, 622, A12

Heckman, T. M. \& Best, P. N. 2014, ARA\&A, 52, 589

Hogan, M. T., Edge, A. C., Hlavacek-Larrondo, J., et al. 2015, MNRAS, 453, 1201

Huchra, J. P., Macri, L. M., Masters, K. L., et al. 2012, ApJS, 199, 26

Hudson, D. S., Mittal, R., Reiprich, T. H., et al. 2010, A\&A, 513, A37

Ineson, J., Croston, J. H., Hardcastle, M. J., et al. 2015, MNRAS, 453, 2682

Intema, H. T., Jagannathan, P., Mooley, K. P., \& Frail, D. A. 2017, A\&A, 598, A78

Jarvis, M., Taylor, R., Agudo, I., et al. 2016, in MeerKAT Science: On the Pathway to the SKA, 6

Jetha, N. N., Ponman, T. J., Hardcastle, M. J., \& Croston, J. H. 2007, MNRAS, 376, 193

Johnson, R., Ponman, T. J., \& Finoguenov, A. 2009, MNRAS, 395, 1287

Kelly, B. C. 2007, ApJ, 665, 1489

Klein, M., Grandis, S., Mohr, J. J., et al. 2019, MNRAS, 488, 739

Klein, M., Mohr, J. J., Desai, S., et al. 2018, MNRAS, 474, 3324

Klein, M., Oguri, M., Mohr, J. J., et al. 2021, A\&A, (accepted)

Kolokythas, K., O'Sullivan, E., Intema, H., et al. 2019, MNRAS, 489, 2488

Kolokythas, K., O’Sullivan, E., Raychaudhury, S., et al. 2018, MNRAS, 481, 1550

Ledlow, M. J., Owen, F. N., \& Eilek, J. A. 2002, New A Rev., 46, 343

Liu, A., Bulbul, E., Ghirardini, V., et al. 2021, A\&A, (submitted)

Liu, A., Zhai, M., \& Tozzi, P. 2019, MNRAS, 485, 1651

Lovisari, L., Forman, W. R., Jones, C., et al. 2017, ApJ, 846, 51

Lovisari, L., Reiprich, T. H., \& Schellenberger, G. 2015, A\&A, 573, A118

Lovisari, L., Schellenberger, G., Sereno, M., et al. 2020, ApJ, 892, 102

Main, R. A., McNamara, B. R., Nulsen, P. E. J., Russell, H. R., \& Vantyghem, A. N. 2017, MNRAS, 464, 4360

Malarecki, J. M., Jones, D. H., Saripalli, L., Staveley-Smith, L., \& Subrahmanyan, R. 2015, MNRAS, 449, 955

Markevitch, M. \& Vikhlinin, A. 2007, Phys. Rep., 443, 1

McDonald, M., McNamara, B. R., Voit, G. M., et al. 2019, ApJ, 885, 63

McNamara, B. R. \& Nulsen, P. E. J. 2007, ARA\&A, 45, 117

McNamara, B. R. \& Nulsen, P. E. J. 2012, New Journal of Physics, 14, 055023

McNamara, B. R., Wise, M., Nulsen, P. E. J., et al. 2000, ApJ, 534, L135

Merloni, A. \& Heinz, S. 2007, MNRAS, 381, 589

Merloni, A., Körding, E., Heinz, S., et al. 2006, New A, 11, 567

Merloni, A., Predehl, P., Becker, W., et al. 2012, ArXiv e-prints [arXiv: 1209.3114]

Mittal, R., Hudson, D. S., Reiprich, T. H., \& Clarke, T. 2009, A\&A, 501, 835

Mohan, N. \& Rafferty, D. 2015, PyBDSF: Python Blob Detection and Source Finder

Moravec, E., Gonzalez, A. H., Stern, D., et al. 2020, ApJ, 888, 74

Morganti, R., Fogasy, J., Paragi, Z., Oosterloo, T., \& Orienti, M. 2013, Science, 341, 1082

O'Sullivan, E., Giacintucci, S., David, L. P., et al. 2011a, ApJ, 735, 11

O'Sullivan, E., Giacintucci, S., David, L. P., Vrtilek, J. M., \& Raychaudhury, S. 2011b, MNRAS, 411, 1833

O'Sullivan, E., Ponman, T. J., Kolokythas, K., et al. 2017, MNRAS, 472, 1482

Padovani, P., Alexander, D. M., Assef, R. J., et al. 2017, A\&A Rev., 25, 2 
Pasini, T., Brüggen, M., de Gasperin, F., et al. 2020, MNRAS, 497, 2163

Pasini, T., Finoguenov, A., Brüggen, M., et al. 2021a, MN-

RAS[arXiv: 2105.08727]

Pasini, T., Gitti, M., Brighenti, F., et al. 2021b, ApJ, 911, 66

Pasini, T., Gitti, M., Brighenti, F., et al. 2019, ApJ, 885, 111

Predehl, P., Andritschke, R., Arefiev, V., et al. 2021, A\&A, 647, A1

Rafferty, D. A., McNamara, B. R., Nulsen, P. E. J., \& Wise, M. W. 2006, ApJ, 652,216

Rasia, E., Borgani, S., Murante, G., et al. 2015, ApJ, 813, L17

Roger, R. S., Costain, C. H., \& Bridle, A. H. 1973, AJ, 78, 1030

Rossetti, M., Gastaldello, F., Ferioli, G., et al. 2016, MNRAS, 457, 4515

Rossetti, M. \& Molendi, S. 2010, A\&A, 510, A83

Sabater, J., Best, P. N., \& Argudo-Fernández, M. 2013, MNRAS, 430, 638

Sabater, J., Best, P. N., Hardcastle, M. J., et al. 2019, A\&A, 622, A17

Schinnerer, E., Sargent, M. T., Bondi, M., et al. 2010, ApJS, 188, 384

Shen, L., Miller, N. A., Lemaux, B. C., et al. 2017, MNRAS, 472, 998

Shimwell, T. W., Röttgering, H. J. A., Best, P. N., et al. 2017, A\&A, 598, A104

Shimwell, T. W., Tasse, C., Hardcastle, M. J., et al. 2019, A\&A, 622, A1

Sijacki, D., Vogelsberger, M., Genel, S., et al. 2015, MNRAS, 452, 575

Smirnov, O. M. \& Tasse, C. 2015, MNRAS, 449, 2668

Smolčić, V., Novak, M., Bondi, M., et al. 2017, A\&A, 602, A1

Soker, N. \& Pizzolato, F. 2005, ApJ, 622, 847

Sun, M. 2009, ApJ, 704, 1586

Sun, M. 2012, New Journal of Physics, 14, 045004

Sun, M., Jones, C., Forman, W., et al. 2007, ApJ, 657, 197

Tasse, C. 2014a, arXiv, 1

Tasse, C. 2014b, A\&A, 566, A127

Tasse, C., Hugo, B., Mirmont, M., et al. 2018, A\&A, 611, 1

Tasse, C., Shimwell, T., Hardcastle, M. J., et al. 2021, A\&A, 648, A1

Ubertosi, F., Gitti, M., Torresi, E., Brighenti, F., \& Grandi, P. 2021, MNRAS

van Haarlem, M. P., Wise, M. W., Gunst, A. W., et al. 2013, A\&A, 556, A2

van Weeren, R. J., Williams, W. L., Hardcastle, M. J., et al. 2016, Astrophys. J. Suppl. Ser., 223, 2

Vikhlinin, A., Kravtsov, A., Forman, W., et al. 2006, ApJ, 640, 691

Von Der Linden, A., Best, P. N., Kauffmann, G., \& White, S. D. M. 2007, MNRAS, 379,867

Werner, N., Oonk, J. B. R., Sun, M., et al. 2014, MNRAS, 439, 2291

White, R. L., Becker, R. H., Helfand, D. J., \& Gregg, M. D. 1997, ApJ, 475, 479

Williams, W. L., van Weeren, R. J., Röttgering, H. J. A., et al. 2016, MNRAS, 460, 2385

Willis, J. P., Pacaud, F., Valtchanov, I., et al. 2005, MNRAS, 363, 675

Willott, C. J., Rawlings, S., Blundell, K. M., \& Lacy, M. 1999, MNRAS, 309, 1017 


\section{Appendix A: Examples of interesting systems}

The high flux sensitivity and spatial coverage of eROSITA and LOFAR at their respective frequencies allows for interesting comparisons. In the past, the combination of X-ray and radio observations of galaxy clusters and of their BCGs have led to a significant improvement in the understanding of the thermal and non-thermal processes in these environments (e.g. Gitti et al. 2010; Kolokythas et al. 2018; Botteon et al. 2020; see Sec. 1 for more references and reviews).

We used the eROSITA and LOFAR observations to look for systems showing interesting morphologies and signs of possible interaction between the ICM and the central AGN. In this section, we present four among the most interesting examples of such clusters. We focus on AGN emission only, while diffuse emission more directly associated with the ICM and clusters dynamical state will be presented in a forthcoming paper (Hoang et al. in prep.). Table A.1 summarises the main properties of these systems.

\section{Appendix A.0.1: eFEDSJ085022.3+001607}

eFEDSJ085022.3+001607 (left panel of Fig. A.1) is located at a redshift of $z=0.196$ (spectroscopic). The strongly elliptical and irregular morphology of the X-ray emission and low concentration $\left(c_{\mathrm{SB}}=0.02\right)$ suggest that this cluster is disturbed. The BCG hosts an elongated, head-tail shaped radio galaxy (major axis $500 \mathrm{kpc})$, with a $144 \mathrm{MHz}$ luminosity of $L_{R}=(4.1 \pm 0.2) \times 10^{24}$ $\mathrm{W} \mathrm{Hz}^{-1}$. The AGN lies at $\sim 150 \mathrm{kpc}$ from the X-ray peak. Surface brightness discontinuities that coincide with the lobes of the radio galaxy are detected in the X-ray image. However, the relatively low resolution does not reveal any ICM cavities, which however have never been detected around head-tails. The shape of the non-thermal emission follows that of the hot plasma, with the jet extending towards the East through the X-ray ripple. Meanwhile, the expansion in the opposite direction appears frustrated.

\section{Appendix A.0.2: eFEDSJ085830.1-010656}

The irregular morphology and low concentration $\left(c_{\mathrm{SB}}=0.13\right)$ of eFEDSJ085830.1-010656 (right panel of Fig. A.1) leads us to classify it as a non cool core. The BCG hosts a wide angle tail radio galaxy with two tails departing in the $\mathrm{S}$ and $\mathrm{SW}$ directions for $\sim 250 \mathrm{kpc}$ each. The tails are expanding into a lower-density region within the group. Deeper X-ray observations are needed to study the ICM emission of this group due to its low surface brightness and relatively high redshift.

\section{Appendix A.0.3: eFEDSJ091322.9+040618}

eFEDSJ091322.9+040618 (left panel of Fig. A.2) is a lowredshift $(z=0.088$, spectroscopic) galaxy group classified as a disturbed cluster due to its irregular shape and low concentration $\left(c_{\mathrm{SB}}=0.04\right)$. The radio galaxy extends for more than $200 \mathrm{kpc}$ along the NW-SE axis. The lobes are expanding into the SE and NW directions following the hot plasma. Diffuse emission with unclear origin is detected in the SE direction, correspondingly to a low surface brightness region, extending for $\sim 150 \mathrm{kpc}$.

\section{Appendix A.0.4: eFEDSJ093056.9+034826}

eFEDSJ093056.9+034826 (right panel of Fig. A.2) is a galaxy group located at $z=0.09$ (photometric). The elliptical shape and relatively high concentration $\left(c_{\mathrm{SB}}=0.21\right)$ classify it as a moderate cool core. The BCG hosts a double-lobe elongated radio galaxy with a major axis of $\sim 600 \mathrm{kpc}$ and $L_{R}=(7.7 \pm 0.2) \times 10^{23}$ $\mathrm{W} \mathrm{Hz}{ }^{-1}$. The long lobes $(\sim 300 \mathrm{kpc})$ of the central radio galaxy are extending far beyond the $\mathrm{X}$-ray bright core of the group. The low X-ray flux of this group makes it difficult to identify depressions in the surface brightness. 

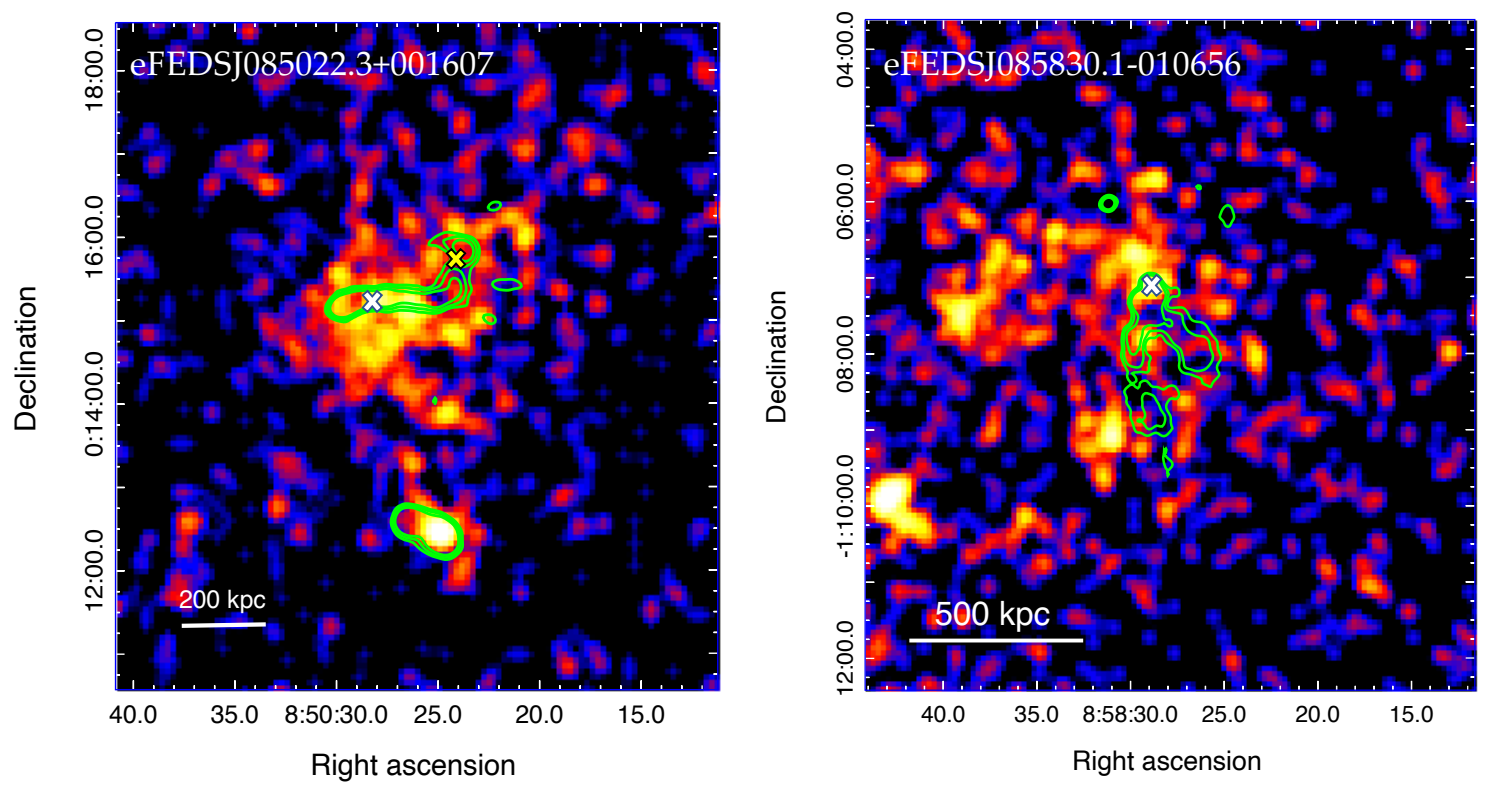

Fig. A.1. eROSITA 0.2-2.3 keV images of eFEDSJ085022.3+001607 (left panel) and eFEDSJ085830.1-010656 (right panel), smoothed with a $3 \sigma$ gaussian filter. LOFAR $144 \mathrm{MHz}$ contours at 3,6,12,24 $\cdot \mathrm{rms}$ (local) are in green. The white cross represents the cluster X-ray peak, while the yellow cross is the BCG position. For eFEDSJ085830.1-010656, the BCG is coincident with the X-ray peak.
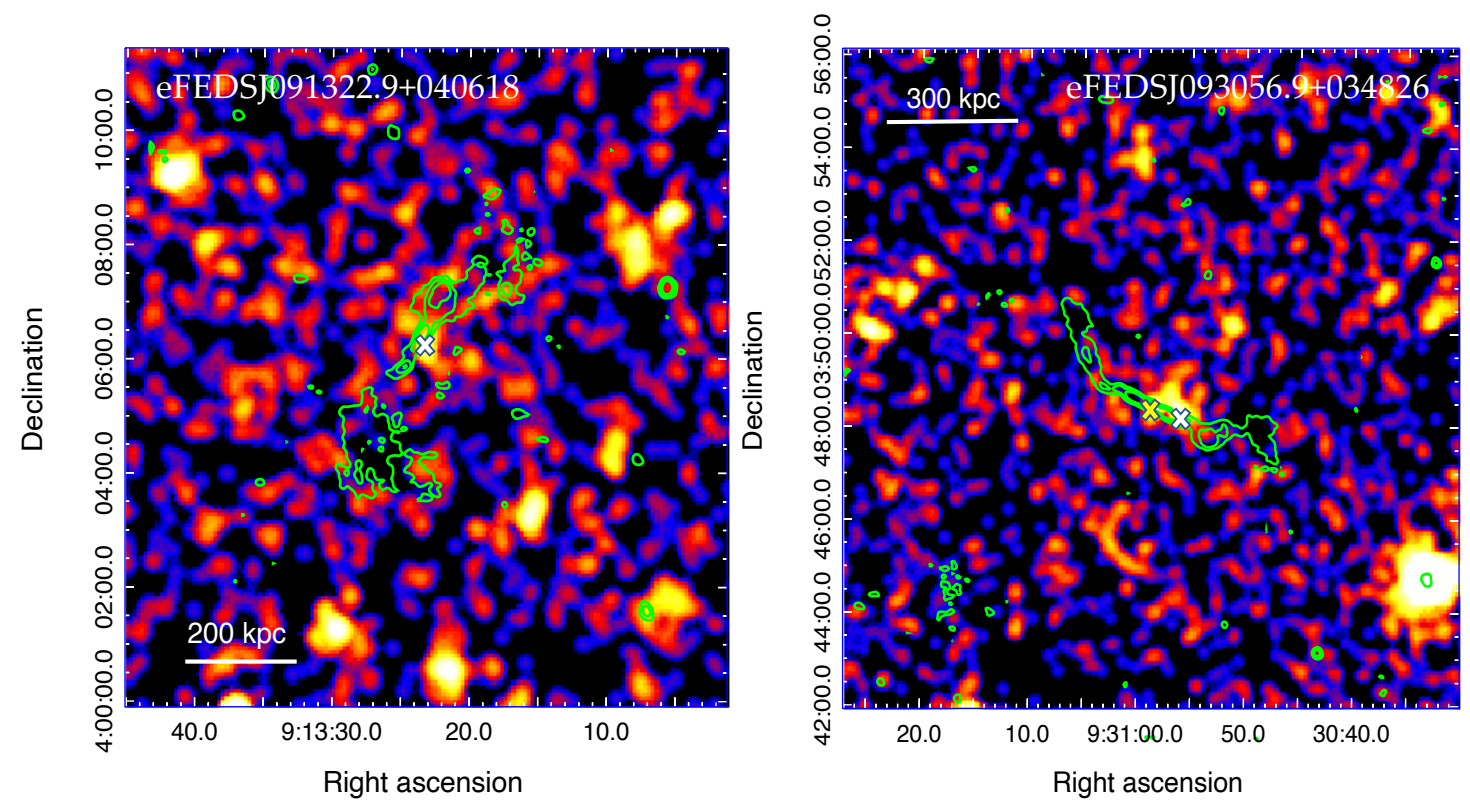

Fig. A.2. eROSITA 0.2-2.3 keV images of eFEDSJ091322.9+040618 (left panel) and eFEDSJ093056.f9+034826 (right panel), smoothed with a $3 \sigma$ gaussian filter. LOFAR $144 \mathrm{MHz}$ contours at 3,6,12,24 $\cdot \mathrm{rms}$ (local) are in green. The white cross represents the cluster X-ray peak. For eFEDSJ091322.9+040618, the BCG is coincident with the X-ray peak.

Table A.1. X-ray observables and BCG radio power for 4 relevant eFEDS clusters

\begin{tabular}{cccccc}
\hline \hline Name & $z$ & $k T^{a}[\mathrm{keV}]$ & $L_{\text {bol }}^{a}\left[10^{43} \mathrm{erg} \mathrm{s}^{-1}\right]$ & $c_{\mathrm{SB}}$ & $L_{R}^{b}\left[10^{24} \mathrm{~W} \mathrm{~Hz}^{-1}\right]$ \\
\hline eFEDSJ085022.3+001607 & 0.196 & $3.1 \pm_{0.7}^{1.1}$ & $2.7 \pm_{0.9}^{1.3}$ & 0.02 & $4.1 \pm 0.2$ \\
eFEDSJ085830.1-010656 & 0.224 & $2.1 \pm_{0.8}^{1.7}$ & $2.1 \pm_{0.4}^{0.5}$ & 0.13 & $25.0 \pm 1.0$ \\
eFEDSJ091322.9+040618 & 0.088 & $0.45 \pm_{0.17}^{0.29}$ & $4.1 \pm_{0.9}^{1.2}$ & 0.04 & $0.74 \pm 0.05$ \\
eFEDSJ093056.9+034826 & 0.09 & $0.61 \pm_{0.27}^{0.75}$ & $2.7 \pm_{0.9}^{1.2}$ & 0.21 & $0.77 \pm 0.02$ \\
\hline \hline
\end{tabular}

Notes: ${ }^{a}$ : estimated within $500 \mathrm{kpc}^{b}$ : $144 \mathrm{MHz}$ luminosity of the BCG. 